\title{
Diversity and distribution of arid-semi arid truffle (Terfezia and Picoa) in Elazı̆g-Malatya region of Turkey
}

\author{
Akyüz $\mathbf{M}^{1^{*}}$, Kırbağ $S^{2}$, Bircan $B^{2}$ and Gürhan $Y^{1}$ \\ ${ }^{1}$ Department of Biology, Faculty of Science and Arts, Bitlis Eren University, Bitlis - TURKEY \\ ${ }^{2}$ Department of Biology, Faculty of Science, Firat University, Elazı
}

Akyüz M, Kırbağ S, Bircan B, Gürhan Y 2015 - Diversity and distribution of arid-semi arid truffle (Terfezia and Picoa) in Elazı̆̆-Malatya region of Turkey. Mycosphere 6(6), 766-783, Doi 10.5943/mycosphere/6/6/11

\begin{abstract}
This work aims to conduct research for finding arid-semi arid (desert) truffles that grow in the Elazığ-Malatya area. It was determined that Picoa lefebvrei, P. juniperi, Terfezia olbiensis, T. claveryi and $T$. boudieri grew naturally in the study areas. Short descriptions of the taxon are provided together with the photograps of fruit bodies and macro-microstructures. In addition, we present new localities for Picoa lefebvrei, P. juniperi, Terfezia olbiensis, T. claveryi and T. boudieri in Turkey. The habitats of these species should be protected by turning the growing fields into natural protected areas.
\end{abstract}

Key words - Ascomycotina - arid-semi arid truffle - biodiversity - Helianthemum spp. hypogeous - Picoa - Terfezia

\section{Introduction}

Turkey has one of the richest macrofungi floras in the northern hemisphere as it is located at the convergence of three phytogeographical regions: Euro-Siberian, Mediterranean, and IranoTuranian. It has a potentially rich diversity of truffle taxa due to its unique phytogeographical location, climate and soil variability, and vegetation diversity (Castellano \& Türkoğlu 2012, Türkoğlu et al. 2015). Truffles are edible hypogeous fruit bodies produced by many genera of fungi belonging to the class Ascomycetes G.Winter. The hypogeous ascocarps of these fungi are known as truffles. They can be classified as forest truffles, desert truffles, and semi-arid truffles. Among these, Terfezia (Tul. \& C. Tul.) Tul. \& C. Tul. (Pezizaceae Dumort.), Tirmania Chatin (Pezizaceae Dumort.), Picoa Vittad. (Pyronemataceae Corda) and Tuber P. Micheli ex. F.H Wigg (Tuberaceae Dumort.) are classified in different taxa in Pezizales J. Schrot (Roth-Bejerano et al. 2004, Moreno et al. 2014). Underground members of the Pezizaceae are well distributed around the globe. Arid and semi arid truffles are adapted to exploit different types of soil in association with specific hosts (Diez et al. 2002). Terfezia, Tirmania and Picoa species form mycorrhizae mainly on roots of the family Cistaceae, including different species of the genus Helianthemum (L.) Miller (Mandeel \& Al-Laith 2007, Kagan-Zur \& Roth-Bejerano 2008, Kovacs et al. 2011, Chevalier 2014), as well as other symbionts.

Every country has the responsibility to determine its biological richnesses, protect their habitats, and ensure the continuation of the species. Although Turkey is rich in terms of mushroom type diversity, the country cannot make use of these adequately, and in fact, many species are at the 
verge of extinction and under danger.

Little is known about the truffle diversity in Turkey (Türkoğlu \& Castellano 2014), as truffle fungi have received less attention than epigeous fungi. To date, only $\sim 60$ truffle species have been reported from Turkey (Oder 1988, Işıloğlu \& Oder 1995, Afyon 1996, Doğan \& Ozturk 2006, Solak et al. 2007, Kaya 2009, Castellano \& Turkoğlu 2012, Kagan-Zur \& Akyuz 2014, Türkoğlu \& Castellano 2014, Türkoğlu et al. 2015 etc.).

In the present study, Picoa lefebvrei, P. juniperi, Terfezia olbiensis, T. claveryi and $T$. boudieri are identified and added to the knowledge of the truffle flora of Turkey. And also, short descriptions of the taxon are provided together with the photograps of fruit bodies and macromicrostructures. In addition, we present new localities for the previously reported Picoa lefebvrei, $P$. juniperi, Terfezia olbiensis, T. claveryi and T. boudieri.

\section{Materials \& Methods}

Wild samples of fresh Picoa lefebvrei (Pat.) Maire, Picoa juniperi Vittad., Terfezia boudieri Chatin, Terfezia claveryi Chatin and Terfezia olbiensis Tul. \& C. Tul. were collected from Malatya (Central, Kale, Battalgazi, Arguvan Districts and theirs vicinity) and Elazı $\breve{g}$ (Baskil district and its

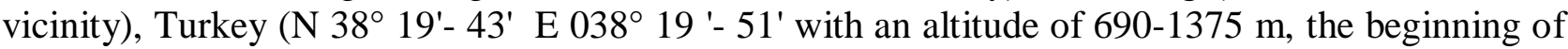
March to the end of May (rarely continue until mid-July), as shown in Fig. 1 and Table 1. They are found on mountain slopes, hilly areas, coastline, roadside, flat areas, steppe, arid-semi arid areas, grow under sandy soil, and appear with spring rains. They are usually collected near Helianthemum salicifolium (L.) Mill. and rarely H. ledifolium (L.) Mill. We identified the location of the truffles from crevices that appeared in the surface of the soil above the truffle. Micromorphological characters were recorded after rehydrating in 3\% KOH or in distilled water (Alsheikh 1994, Montecchi \& Sarasini 2000). Macromorphological characteristics, spores and sterile tissues were photographed with a compound microscope (Olympus SZ61 and CX41). Following standart mycological technigues, necessary macroscopic and microscopic data were obtained. The specimen was identified by examining their macroscobic and microscobic features, using references by Alsheikh \& Trappe (1983), Gücin (1983), Alsheikh (1994), Moreno et al. (2000), Montecchi \& Sarasini (2000), Kirk et al. (2008), Gücin et al. (2010), Castellano \& Türkoğlu (2012), Türkoğlu \& Castellano (2014), Türkoğlu et al. (2015), and Kagan-Zur et al. (2014). The samples were cleaned, cut into slices, dried at room temperature, and then stored.

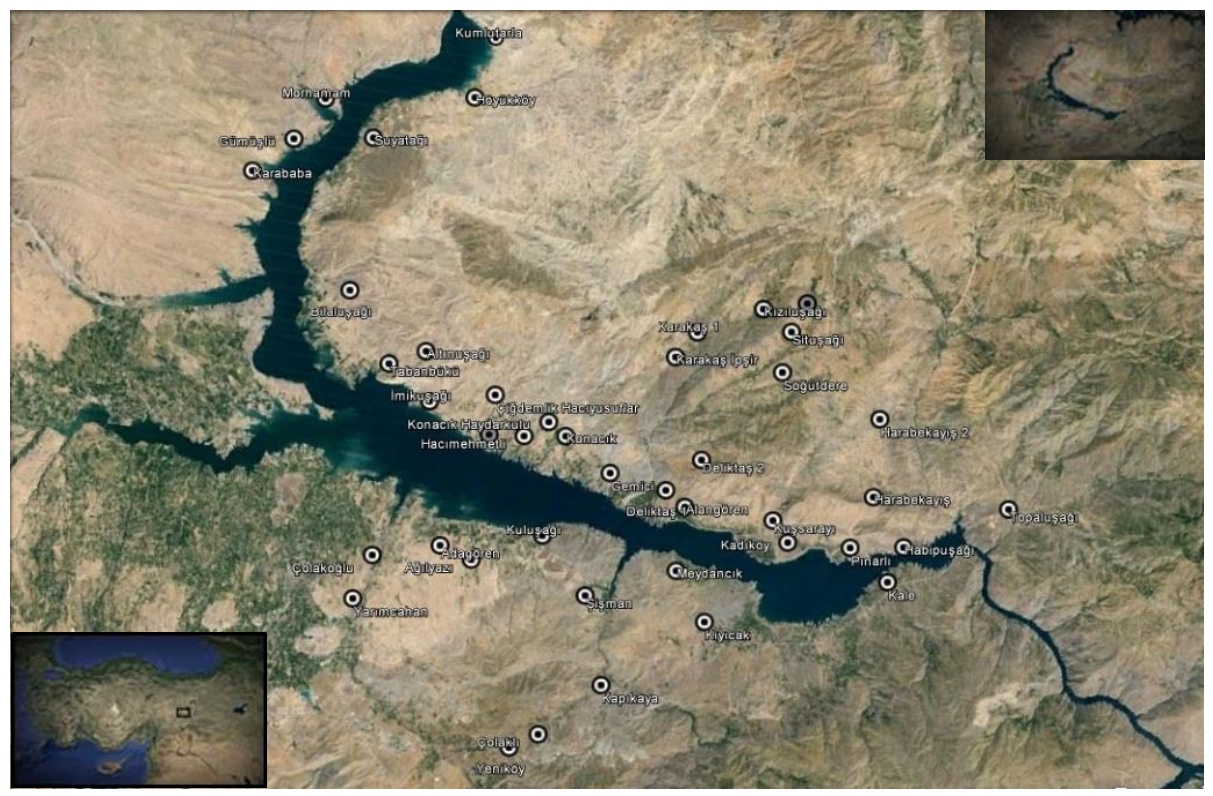

Fig. 1 - Map of the study area (where Malatya (Centre, Kale, Battalgazi, Arguvan districts and their vicinities) and Elazığ (Baskil district and its vicinity) provincial borders intersect, together with the area around Kömürhan Bridge, and at areas along the shores of the Karakaya Dam). 
Table 1 The locations, GPS coordinates, host plant and the location altitudes of the arid and semiarid truffle types that grow in the Elazı $\breve{g}$-Malatya area of Turkey.

\begin{tabular}{|c|c|c|c|c|}
\hline Truffle types & Host plant & Locality & GPS Coordinates & Altitude (m) \\
\hline $\begin{array}{l}\text { T. boudieri } \\
\text { P. lefebvrei } \\
\text { T. boudieri } \\
\text { T.olbiensis } \\
\text { P. lefebvrei }\end{array}$ & H. salicifolium & $\begin{array}{l}\text { Kale and its vicinity, } \\
\text { Kale - Malatya } \\
\text { Kiyıcak village and its vicinity, } \\
\text { Kale - Malatya }\end{array}$ & 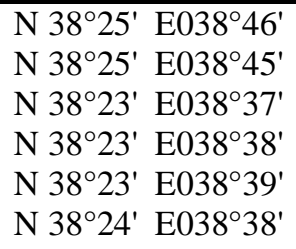 & $698-762$ \\
\hline $\begin{array}{l}\text { T. boudieri } \\
\text { P. lefebvrei }\end{array}$ & H. salicifolium & $\begin{array}{l}\text { Kapıkaya village and its vicinity, } \\
\text { Centre - Malatya }\end{array}$ & 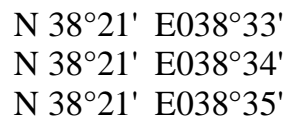 & $756-927$ \\
\hline $\begin{array}{l}\text { T. boudieri } \\
\text { P. lefebvrei }\end{array}$ & H. salicifolium & $\begin{array}{l}\text { Çolaklı village and its vicinity, } \\
\text { Centre - Malatya }\end{array}$ & 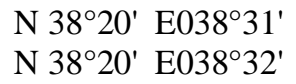 & $913-1089$ \\
\hline $\begin{array}{l}\text { T. boudieri } \\
\text { P. lefebvrei }\end{array}$ & H. salicifolium & $\begin{array}{l}\text { Yeniköy village and its vicinity, } \\
\text { Centre - Malatya }\end{array}$ & 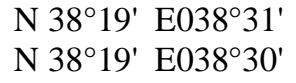 & $1006-1096$ \\
\hline $\begin{array}{l}\text { T. boudieri } \\
\text { T.olbiensis } \\
\text { P. lefebvrei } \\
\text { P. juniperi }\end{array}$ & H. salicifolium & $\begin{array}{l}\text { Meydancik village and its vicinity, } \\
\text { Battalgazi - Malatya }\end{array}$ & $\begin{array}{ll}\text { N } 38^{\circ} 25^{\prime} & \text { E0 } 38^{\circ} 37^{\prime} \\
\text { N } 38^{\circ} 25^{\prime} & \text { E038 } 38^{\prime} \\
\text { N } 38^{\circ} 24^{\prime} & \text { E038 } 38^{\prime}\end{array}$ & $782-895$ \\
\hline $\begin{array}{l}\text { T. boudieri } \\
\text { T.olbiensis } \\
\text { P. lefebvrei }\end{array}$ & H. salicifolium & $\begin{array}{l}\text { Yarımcahan village and its } \\
\text { vicinity, Battalgazi - Malatya }\end{array}$ & 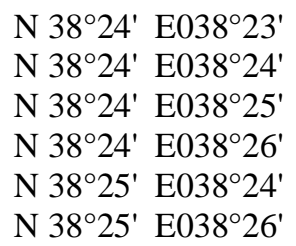 & $753-889$ \\
\hline $\begin{array}{l}\text { T. boudieri } \\
\text { T.olbiensis } \\
\text { P. lefebvrei }\end{array}$ & H. salicifolium & $\begin{array}{l}\text { Çolakoğlu village and its vicinity, } \\
\text { Battalgazi - Malatya }\end{array}$ & $\begin{array}{ll}\text { N } 38^{\circ} 26^{\prime} & \text { E038 } 28^{\circ} \\
\text { N } 38^{\circ} 25^{\prime} & \text { E038 } 25^{\prime} \\
\text { N } 38^{\circ} 25^{\prime} & \text { E038 } 28^{\circ}\end{array}$ & $715-782$ \\
\hline $\begin{array}{l}\text { T. boudieri } \\
\text { T.olbiensis } \\
\text { P. lefebvrei }\end{array}$ & H. salicifolium & $\begin{array}{l}\text { Adagören village and its vicinity, } \\
\text { Battalgazi - Malatya }\end{array}$ & 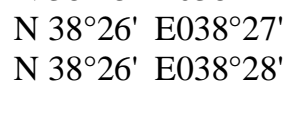 & $706-763$ \\
\hline $\begin{array}{l}\text { T. boudieri } \\
\text { T.olbiensis } \\
\text { P. lefebvrei }\end{array}$ & H. salicifolium & $\begin{array}{l}\text { A ğılyazı village and its vicinity, } \\
\text { Battalgazi - Malatya }\end{array}$ & $\begin{array}{ll}\text { N } 38^{\circ} 26^{\prime} & \text { E038 } 28^{\circ} \\
\text { N } 38^{\circ} 25^{\prime} & \text { E038 } 28^{\prime} \\
\text { N } 38^{\circ} 25^{\prime} & \text { E038 } 29^{\prime}\end{array}$ & $728-808$ \\
\hline $\begin{array}{l}\text { T. boudieri } \\
\text { T.olbiensis } \\
\text { P. lefebvrei }\end{array}$ & H. salicifolium & $\begin{array}{l}\text { Kuluşağı village and its vicinity, } \\
\text { Battalgazi - Malatya }\end{array}$ & 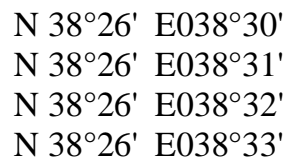 & $703-764$ \\
\hline $\begin{array}{l}\text { T. boudieri } \\
\text { T.olbiensis } \\
\text { P. lefebvrei }\end{array}$ & H. salicifolium & $\begin{array}{l}\text { Şişman village and its vicinity, } \\
\text { Battalgazi - Malatya }\end{array}$ & 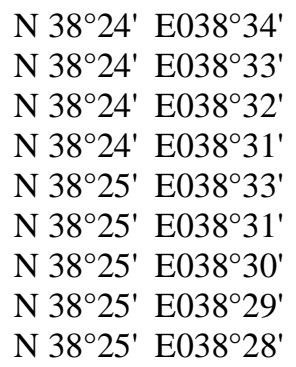 & $748-881$ \\
\hline $\begin{array}{l}\text { T. boudieri } \\
\text { P. lefebvrei }\end{array}$ & H. salicifolium & $\begin{array}{l}\text { Gümüşlü village and its vicinity, } \\
\text { Arguvan - Malatya }\end{array}$ & 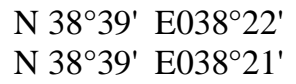 & $705-762$ \\
\hline $\begin{array}{l}\text { T. boudieri } \\
\text { P. lefebvrei }\end{array}$ & H. salicifolium & $\begin{array}{l}\text { Morhamam village and its vicinity, } \\
\text { Arguvan - Malatya }\end{array}$ & $\begin{array}{l}\text { N 38 } 38^{\circ} 41^{\prime} \text { E038 } 22^{\prime} \\
\text { N } 38^{\circ} 40^{\prime} \\
\text { E038 } 23^{\prime}\end{array}$ & $712-754$ \\
\hline $\begin{array}{l}\text { T. boudieri } \\
\text { P. lefebvrei }\end{array}$ & H. salicifolium & $\begin{array}{l}\text { Karababa village and its vicinity, } \\
\text { Arguvan - Malatya }\end{array}$ & 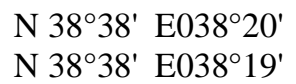 & $719-746$ \\
\hline $\begin{array}{l}\text { T. boudieri } \\
\text { P. lefebvrei }\end{array}$ & H. salicifolium & $\begin{array}{l}\text { Topaluşağı village and its vicinity, } \\
\text { Elazı̆̆ - Malatya provincial } \\
\text { borders, Baskil -Elazığ }\end{array}$ & 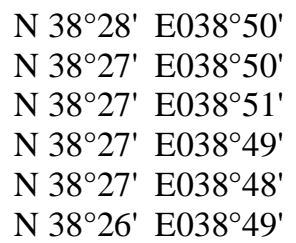 & $691-884$ \\
\hline
\end{tabular}




\begin{tabular}{|c|c|c|c|c|}
\hline Truffle types & Host plant & Locality & GPS Coordinates & Altitude (m) \\
\hline $\begin{array}{l}\text { T. boudieri } \\
\text { P. lefebvrei }\end{array}$ & H. salicifolium & $\begin{array}{l}\text { Habipuşağ village and its vicinity, } \\
\text { Baskil - Elazı̆ }\end{array}$ & 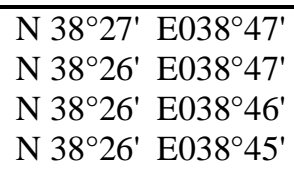 & $696-768$ \\
\hline $\begin{array}{l}\text { T. boudieri } \\
\text { T.olbiensis } \\
\text { P. lefebvrei } \\
\text { P. juniperi }\end{array}$ & H. salicifolium & $\begin{array}{l}\text { Pınarlı village and its vicinity, } \\
\text { Baskil - Elazı } \breve{g}\end{array}$ & 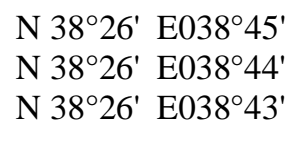 & $699-771$ \\
\hline $\begin{array}{l}\text { T. boudieri } \\
\text { T.olbiensis } \\
\text { P. lefebvrei } \\
\text { P. juniperi }\end{array}$ & H. salicifolium & $\begin{array}{l}\text { Kadıköy village and its vicinity, } \\
\text { Baskil - Elazı̆ }\end{array}$ & 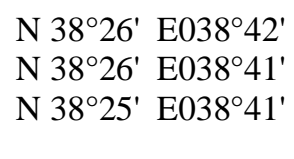 & $704-806$ \\
\hline $\begin{array}{l}\text { T. boudieri } \\
\text { T.olbiensis } \\
\text { P. lefebvrei }\end{array}$ & H. salicifolium & $\begin{array}{l}\text { Kuşsarayı village and its vicinity, } \\
\text { Baskil - Elazı̆ }\end{array}$ & 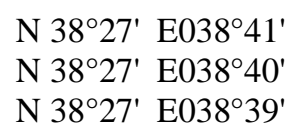 & $777-834$ \\
\hline $\begin{array}{l}\text { T. boudieri } \\
\text { T.olbiensis } \\
\text { P. lefebvrei } \\
\text { P. juniperi }\end{array}$ & H. salicifolium & $\begin{array}{l}\text { Alangören village and its vicinity, } \\
\text { Baskil - Elazığ }\end{array}$ & 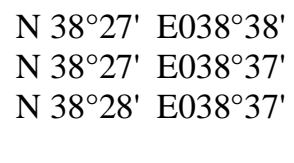 & 736-790 \\
\hline $\begin{array}{l}\text { T. boudieri } \\
\text { T.olbiensis } \\
P . \text { lefebvrei } \\
P \text {. juniperi }\end{array}$ & $\begin{array}{l}\text { H. salicifolium } \\
\text { H. ledifolium }\end{array}$ & $\begin{array}{l}\text { Deliktaş village and its vicinity, } \\
\text { Baskil - Elazı } \breve{g}\end{array}$ & $\begin{array}{ll}\text { N } 38^{\circ} 27^{\prime} & E 038^{\circ} 37^{\prime} \\
\text { N } 38^{\circ} 28^{\prime} & E 038^{\circ} 37^{\prime} \\
\text { N } 38^{\circ} 28^{\prime} & \text { E038 } 38^{\circ} \\
\text { N } 38^{\circ} 29^{\prime} & \text { E038 } 38^{\circ} \\
\text { N } 38^{\circ} 29^{\prime} & \text { E038 } 38^{\circ} \\
\text { N } 38^{\circ} 29^{\prime} & \text { E038 } 38^{\circ}\end{array}$ & $762-1353$ \\
\hline $\begin{array}{l}\text { T. boudieri } \\
\text { T. claveryi } \\
\text { T.olbiensis } \\
\text { P. lefebvrei } \\
\text { P. juniperi }\end{array}$ & H. salicifolium & $\begin{array}{l}\text { Gemici village and its vicinity, } \\
\text { Baskil - Elazığ }\end{array}$ & $\begin{array}{ll}\text { N } 38^{\circ} 28^{\prime} & \text { E038 } 38^{\circ} 5^{\prime} \\
\text { N } 38^{\circ} 28^{\prime} & \text { E038 } 34^{\prime} \\
\text { N } 38^{\circ} 29^{\prime} & \text { E038 } 38^{\circ} \\
\text { N } 38^{\circ} 29^{\prime} & \text { E038 } 38^{\circ} \\
\text { N } 38^{\circ} 29^{\prime} & \text { E038 } 33^{\prime}\end{array}$ & $759-818$ \\
\hline $\begin{array}{l}\text { T. boudieri } \\
\text { T.olbiensis } \\
\text { P. lefebvrei }\end{array}$ & H. salicifolium & $\begin{array}{l}\text { Konacik village, Haydarkulu } \\
\text { hamlet and its vicinity, } \\
\text { Baskil - Elazığ }\end{array}$ & 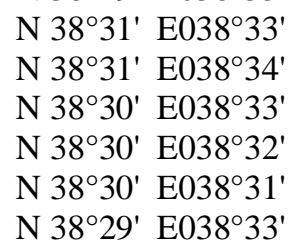 & $762-922$ \\
\hline $\begin{array}{l}\text { T. boudieri } \\
\text { T. claveryi } \\
\text { T.olbiensis } \\
\text { P. lefebvrei } \\
\text { P. juniperi }\end{array}$ & H. salicifolium & $\begin{array}{l}\text { Hacımehmetli village and its } \\
\text { vicinity, Baskil - Elazı } \breve{g}\end{array}$ & $\begin{array}{ll}\text { N } 38^{\circ} 30^{\prime} & \text { E038 } 32^{\circ} \\
\text { N } 38^{\circ} 30^{\prime} & \text { E038 } 31^{\prime} \\
\text { N } 38^{\circ} 29^{\prime} & \text { E038 } 31^{\prime} \\
\text { N } 38^{\circ} 29^{\prime} & \text { E038 } 32^{\prime}\end{array}$ & $728-790$ \\
\hline $\begin{array}{l}\text { T. boudieri } \\
\text { T. claveryi } \\
\text { T.olbiensis } \\
\text { P. lefebvrei } \\
\text { P. juniperi }\end{array}$ & H. salicifolium & $\begin{array}{l}\text { Çiğdemlik village, Haciyusuflar } \\
\text { hamlet and its vicinity, Baskil - } \\
\text { Elazığ }\end{array}$ & 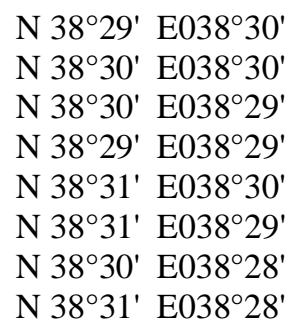 & $701-837$ \\
\hline $\begin{array}{l}\text { T. boudieri } \\
\text { T.olbiensis } \\
\text { P. lefebvrei } \\
\text { P. juniperi }\end{array}$ & $\begin{array}{l}\text { H. salicifolium } \\
\text { H. ledifolium }\end{array}$ & $\begin{array}{l}\text { Karakaş village, İpşir hamlet and } \\
\text { its vicinity, Baskil - Elazı̆̆ }\end{array}$ & $\begin{array}{ll}\text { N } 38^{\circ} 32^{\prime} & E 038^{\circ} 36^{\prime} \\
\text { N } 38^{\circ} 32^{\prime} & E 038^{\circ} 37^{\prime}\end{array}$ & $1115-1260$ \\
\hline $\begin{array}{l}\text { T. boudieri } \\
\text { P. lefebvrei }\end{array}$ & H. salicifolium & $\begin{array}{l}\text { Kızıluşăğ village and its vicinity, } \\
\text { Baskil - Elazığ }\end{array}$ & $\begin{array}{l}\text { N 38 } 38^{\circ} 34^{\prime} \text { E038 } 48^{\prime} \\
\text { N } 38^{\circ} 34^{\prime} \text { E038 } 41^{\prime}\end{array}$ & $1303-1356$ \\
\hline $\begin{array}{l}\text { T. boudieri } \\
\text { P. lefebvrei }\end{array}$ & H. salicifolium & $\begin{array}{l}\text { Yalındam village and its vicinity, } \\
\text { Baskil - Elazı̆g }\end{array}$ & N $38^{\circ} 34^{\prime}$ E038 $42^{\prime}$ & $1320-1375$ \\
\hline $\begin{array}{l}\text { T. boudieri } \\
P . \text { lefebvrei }\end{array}$ & H. salicifolium & $\begin{array}{l}\text { Situşağı village and its vicinity, } \\
\text { Baskil - Elazığ }\end{array}$ & 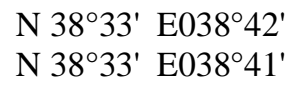 & $1251-1351$ \\
\hline
\end{tabular}




\begin{tabular}{|c|c|c|c|c|}
\hline Truffle types & Host plant & Locality & GPS Coordinates & Altitude (m) \\
\hline T. boudieri & \multirow[t]{4}{*}{ H. salicifolium } & \multirow{4}{*}{$\begin{array}{l}\text { Söğütdere village and its vicinity, } \\
\text { Baskil - Elazı̆g }\end{array}$} & $\mathrm{N} 38^{\circ} 32^{\prime} \mathrm{E} 038^{\circ} 41^{\prime}$ & \multirow[t]{4}{*}{$1174-1210$} \\
\hline T.olbiensis & & & 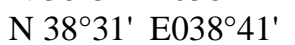 & \\
\hline P. lefebvrei & & & & \\
\hline$P$. juniperi & & & & \\
\hline T. boudieri & \multirow[t]{7}{*}{ H. salicifolium } & \multirow{7}{*}{$\begin{array}{l}\text { Harabekayış village and its vicinity } \\
\text { Baskil - Elazığ }\end{array}$} & $\mathrm{N} 38^{\circ} 28^{\prime} \mathrm{E} 038^{\circ} 45^{\prime}$ & \multirow[t]{7}{*}{$900-1200$} \\
\hline T.olbiensis & & & N $38^{\circ} 28^{\prime}$ E038⒋ & \\
\hline P. lefebvrei & & & N $38^{\circ} 27^{\prime}$ E038 $45^{\prime}$ & \\
\hline$P$. juniperi & & & N $38^{\circ} 27^{\prime}$ E038⒋ & \\
\hline & & & N $38^{\circ} 29^{\prime}$ E038 $45^{\prime}$ & \\
\hline & & & N 3830' E038 $44^{\prime}$ & \\
\hline & & & N $38^{\circ} 30^{\prime}$ E0384' & \\
\hline T. boudieri & \multirow[t]{4}{*}{ H. salicifolium } & \multirow{4}{*}{$\begin{array}{l}\text { İmikuşağ village and its vicinity, } \\
\text { Baskil - Elazı̆g }\end{array}$} & 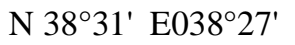 & \multirow[t]{4}{*}{$724-812$} \\
\hline T.olbiensis & & & N $38^{\circ} 30^{\prime} \mathrm{E} 038^{\circ} 28^{\prime}$ & \\
\hline P. lefebvrei & & & & \\
\hline P. juniperi & & & & \\
\hline T. boudieri & \multirow[t]{4}{*}{ H. salicifolium } & \multirow{4}{*}{$\begin{array}{l}\text { Tabanbükü village and its vicinity } \\
\text { Baskil - Elazığ }\end{array}$} & N 383' E038 $25^{\prime}$ & \multirow[t]{4}{*}{$730-812$} \\
\hline T.olbiensis & & & N 3832' E038²5' & \\
\hline P. lefebvrei & & & N $38^{\circ} 33^{\prime}$ E03825' & \\
\hline$P$. juniperi & & & & \\
\hline T. boudieri & \multirow{4}{*}{ H. salicifolium } & \multirow{4}{*}{$\begin{array}{l}\text { Altınuşă̆ } \breve{g}_{1} \text { Village and its vicinity, } \\
\text { Baskil - Elazığ }\end{array}$} & 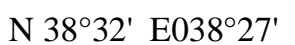 & \multirow{4}{*}{$800-950$} \\
\hline T.olbiensis & & & N $38^{\circ} 32^{\prime}$ E038 $26^{\prime}$ & \\
\hline P. lefebvrei & & & & \\
\hline$P$. juniperi & & & & \\
\hline T. boudieri & \multirow[t]{8}{*}{ H. salicifolium } & \multirow{8}{*}{$\begin{array}{l}\text { Bilaluşağı village and its vicinity, } \\
\text { Baskil - Elazı } \breve{g}\end{array}$} & N 383' E038 24' & \multirow[t]{8}{*}{$722-893$} \\
\hline T. claveryi & & & N 383' E038²4' & \\
\hline T.olbiensis & & & N $38^{\circ} 34^{\prime} \mathrm{E} 038^{\circ} 23^{\prime}$ & \\
\hline P. lefebvrei & & & N $38^{\circ} 35^{\prime}$ E0382' & \\
\hline$P$. juniperi & & & N $38^{\circ} 36^{\prime}$ E $038^{\circ} 23^{\prime}$ & \\
\hline & & & N $38^{\circ} 36^{\prime} \mathrm{E} 038^{\circ} 22^{\prime}$ & \\
\hline & & & 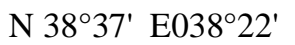 & \\
\hline & & & N $38^{\circ} 37^{\prime} \mathrm{E} 038^{\circ} 23^{\prime}$ & \\
\hline T. boudieri & \multirow[t]{6}{*}{ H. salicifolium } & \multirow{6}{*}{$\begin{array}{l}\text { Suyatağı village and its vicinity, } \\
\text { Baskil - Elazığ }\end{array}$} & N 3839' E038²4' & \multirow[t]{6}{*}{$690-815$} \\
\hline P. lefebvrei & & & N $38^{\circ} 40^{\prime}$ E038⒉ & \\
\hline & & & N 384ㅇ' E038 $24^{\prime}$ & \\
\hline & & & N 3839' E03825' & \\
\hline & & & N 38 $38^{\circ}$ E $038^{\circ} 23^{\prime}$ & \\
\hline & & & N $38^{\circ} 37^{\prime} \mathrm{E} 038^{\circ} 23^{\prime}$ & \\
\hline T. boudieri & \multirow[t]{3}{*}{ H. salicifolium } & \multirow{3}{*}{$\begin{array}{l}\text { Höyükköy village and its vicinity, } \\
\text { Baskil - Elazığ }\end{array}$} & N 38 $41^{\prime}$ E038 $29^{\prime}$ & \multirow[t]{3}{*}{$709-740$} \\
\hline P. lefebvrei & & & N 384ㅇ' E038 $29^{\prime}$ & \\
\hline & & & N $38^{\circ} 40^{\prime} \mathrm{E} 038^{\circ} 28^{\prime}$ & \\
\hline T. boudieri & \multirow[t]{2}{*}{ H. salicifolium } & Kumlutarla village and its vicinity, & 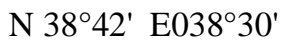 & $702-750$ \\
\hline P. lefebvrei & & Baskil - Elazı̆̆ & 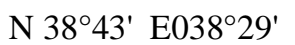 & \\
\hline
\end{tabular}

\section{Results}

Kingdom: Myceteae

Classis: Ascomycetes

Pyronemataceae Corda.

Picoa Vittad.

Picoa lefebvrei (Pat.) Maire

Malatya city Central district (are found in the areas surrounding Kap1kaya village and its

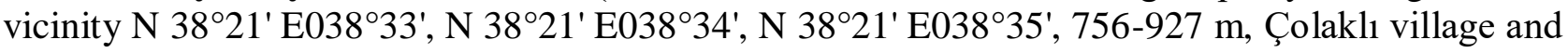
its vicinity N $38^{\circ} 20^{\prime} \mathrm{E} 038^{\circ} 31^{\prime}, \mathrm{N}_{3}^{\circ} 20^{\circ} \mathrm{E} 038^{\circ} 32^{\prime}, 913-1089 \mathrm{~m}$, Yeniköy village and its vicinity $\mathrm{N}$ $38^{\circ} 19^{\prime} \mathrm{E} 038^{\circ} 31^{\prime}, \mathrm{N} 38^{\circ} 19^{\prime} \mathrm{E} 038^{\circ} 30^{\prime}, 1006-1096 \mathrm{~m}$ ), Kale district (are found in the areas

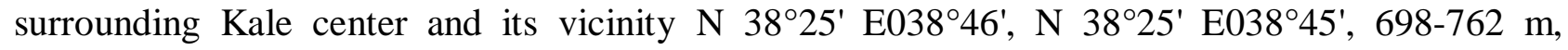

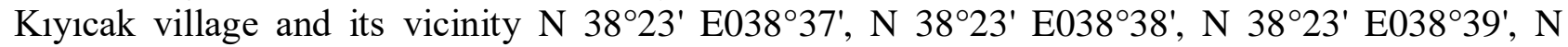
$38^{\circ} 24^{\prime} \mathrm{E} 038^{\circ} 38^{\prime}, 729-886 \mathrm{~m}$ ), Battalgazi district (are found in the areas surrounding Meydanc1k

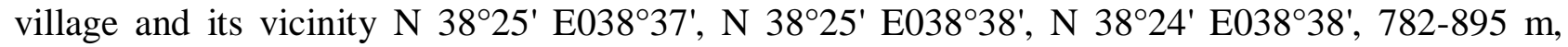




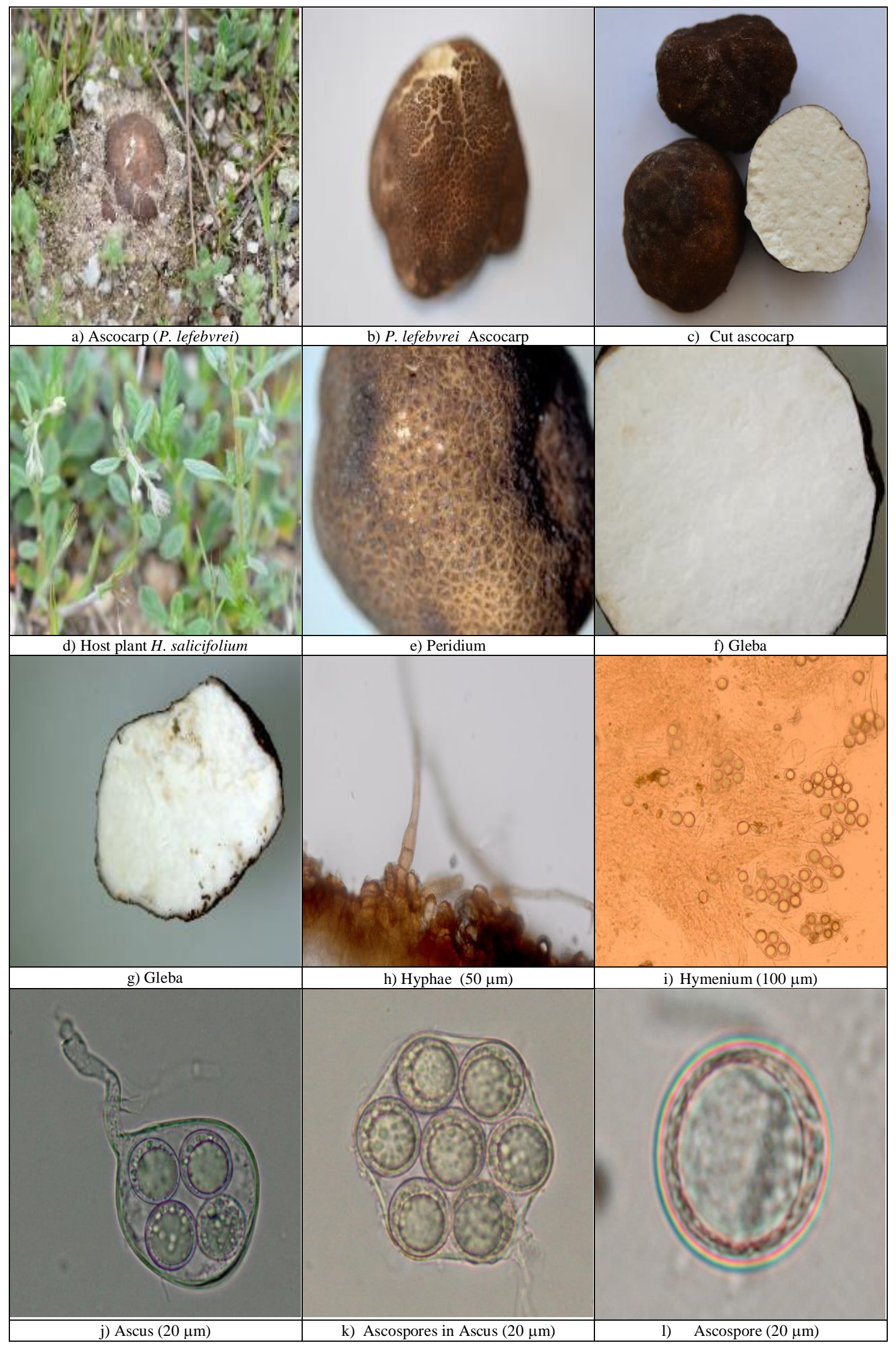

Fig. 2 - The macroscopic and microscopic characteristics (ascocarp, host plant, peridium, gleba, hyphae, hymenium, ascus and ascospore) of P. lefebvrei that were found in the Elaziğ - Malatya area. 


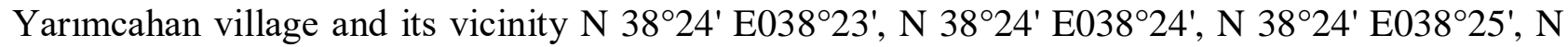

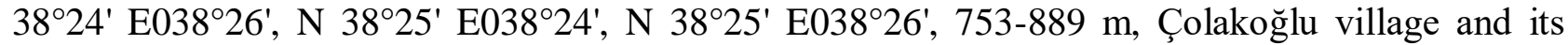

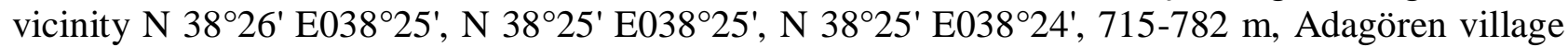
and its vicinity N $38^{\circ} 26^{\prime} \mathrm{E} 038^{\circ} 27^{\prime}, \mathrm{N} 38^{\circ} 26^{\prime} \mathrm{E} 038^{\circ} 28^{\prime}, 706-763 \mathrm{~m}$, Ağ 1 lyazı village and its vicinity

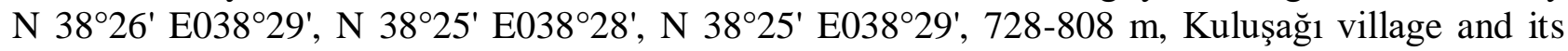

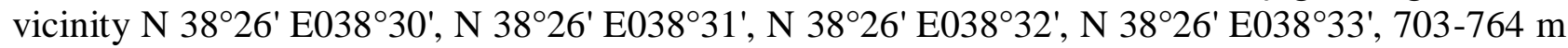

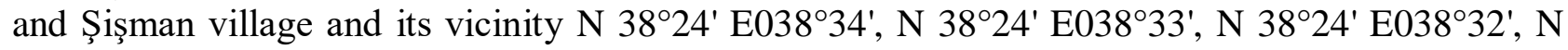

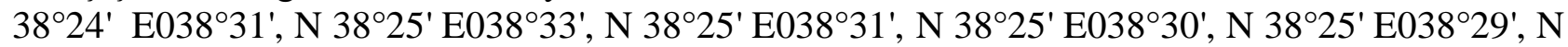
$38^{\circ} 25^{\prime}$ E038 $28^{\circ}, 748-881 \mathrm{~m}$ ) and Arguvan district (are found in the areas surrounding Gümüşlü village and its vicinity N 38 $39^{\prime}$ E038 $22^{\prime}$, N 38 $39^{\prime}$ E038 $21^{\prime}$, 705-762 m, Morhamam village and its vicinity N $38^{\circ} 41^{\prime} \mathrm{E} 038^{\circ} 22^{\prime}$, N 38 $48^{\circ}$ E038 $23^{\circ}, 712-754 \mathrm{~m}$ and Karababa village and its vicinity $\mathrm{N} 38^{\circ} 38^{\prime} \mathrm{E} 038^{\circ} 20^{\prime}, \mathrm{N} 38^{\circ} 38^{\prime} \mathrm{E} 038^{\circ} 19^{\prime}, 719-746 \mathrm{~m}$ ). It was determined that $H$. salicifolium, which is its mycorrhiza, grows naturally from March to May (Table 1, Fig. 2). Elazı̆ city Baskil district (are found in the areas surrounding Topaluşağ 1 village and its vicinity N $38^{\circ} 28^{\prime} \mathrm{E} 038^{\circ} 50^{\prime}, \mathrm{N} 38^{\circ} 27^{\prime}$

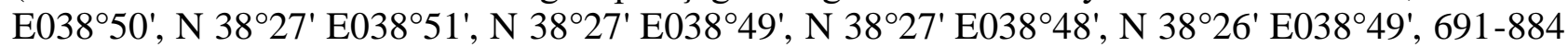

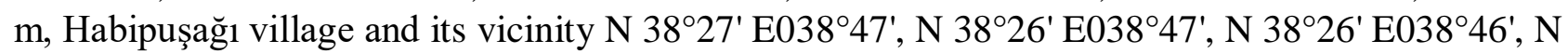
$38^{\circ} 26^{\prime} \mathrm{E} 038^{\circ} 45^{\prime}, 696-768 \mathrm{~m}$, P1narli village and its vicinity N 38 $26^{\prime} \mathrm{E} 038^{\circ} 45^{\prime}, \mathrm{N} 38^{\circ} 26^{\prime} \mathrm{E} 038^{\circ} 44^{\prime}$,

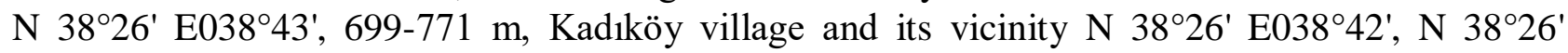

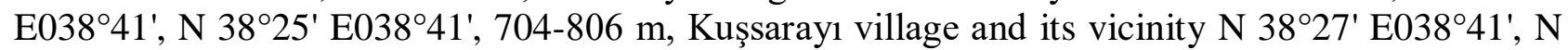
$38^{\circ} 27^{\prime} \mathrm{E} 038^{\circ} 40^{\prime}, \mathrm{N} 38^{\circ} 27^{\prime} \mathrm{E} 038^{\circ} 39^{\prime}, 777-834 \mathrm{~m}$, Alangören village and its vicinity N $38^{\circ} 27^{\prime}$ E038 $38^{\prime}, \mathrm{N} 38^{\circ} 27^{\prime} \mathrm{E} 038^{\circ} 37^{\prime}, \mathrm{N} 38^{\circ} 28^{\prime} \mathrm{E} 038^{\circ} 37^{\prime}, 736-790 \mathrm{~m}$, Deliktaş village and its vicinity $\mathrm{N}$

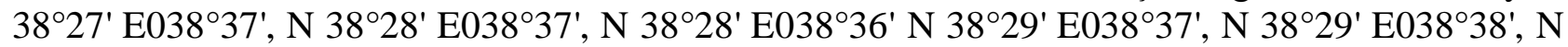

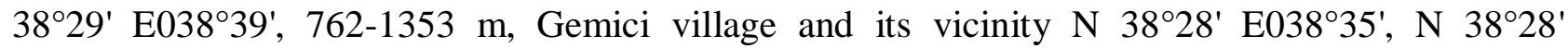

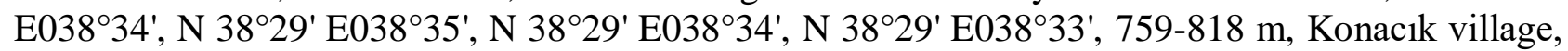

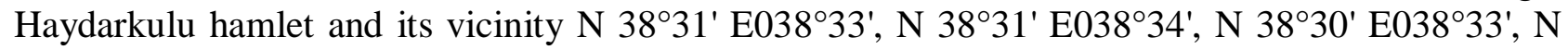
$38^{\circ} 30^{\prime} \mathrm{E} 038^{\circ} 32^{\prime}, \mathrm{N} 38^{\circ} 30^{\prime} \mathrm{E} 038^{\circ} 31^{\prime}, \mathrm{N} 38^{\circ} 29^{\prime} \mathrm{E} 038^{\circ} 33^{\prime}$, 762-922 m, Hacimehmetli village and its

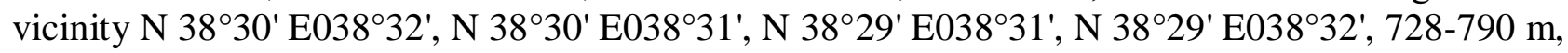

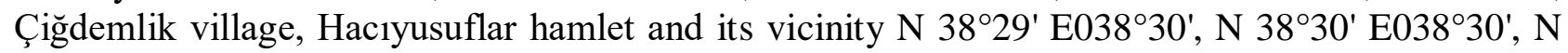
$38^{\circ} 30^{\prime} \mathrm{E} 038^{\circ} 29^{\prime}, \mathrm{N} 38^{\circ} 29^{\prime} \mathrm{E} 038^{\circ} 29^{\prime}, \mathrm{N} 38^{\circ} 31^{\prime} \mathrm{E} 038^{\circ} 30^{\prime}, \mathrm{N} 38^{\circ} 31^{\prime} \mathrm{E} 038^{\circ} 29^{\prime}, \mathrm{N} 38^{\circ} 30^{\prime} \mathrm{E} 038^{\circ} 28^{\prime}, \mathrm{N}$ $38^{\circ} 31^{\prime} \mathrm{E} 038^{\circ} 28^{\prime}, 701-837 \mathrm{~m}$, Karakaş village, İpşir hamlet and its vicinity N 38 $32^{\prime}$ E038 $36^{\prime}$, N

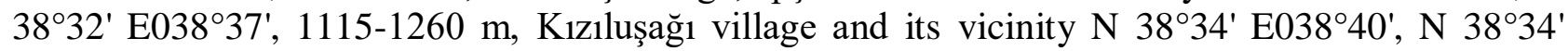
E038 $41^{\prime}$ 1303-1356 m, Yalındam village and its vicinity $\mathrm{N} 38^{\circ} 34^{\prime}$ E038 $42^{\prime}, 1320-1375 \mathrm{~m}$,

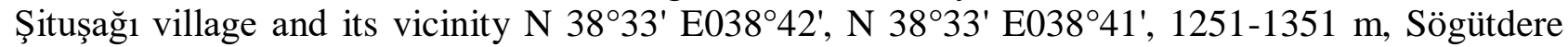
village and its vicinity N $38^{\circ} 31^{\prime} \mathrm{E} 038^{\circ} 41^{\prime}, \mathrm{N} 38^{\circ} 32^{\prime} \mathrm{E} 038^{\circ} 41^{\prime}, 1174-1210 \mathrm{~m}$, Harabekayıs village

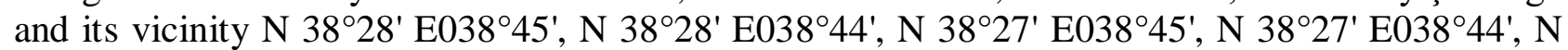
$38^{\circ} 29^{\prime}$ E038 $45^{\prime}, \mathrm{N} 38^{\circ} 30^{\prime} \mathrm{E} 038^{\circ} 44^{\prime}, \mathrm{N} 38^{\circ} 30^{\prime} \mathrm{E} 038^{\circ} 45^{\prime}$, 900-1200 m, İmikuşağ 1 village and its vicinity N $38^{\circ} 31^{\prime}$ E038 $27^{\circ}$, N $38^{\circ} 30^{\prime}$ E038 $28^{\circ}, 724-812 \mathrm{~m}$, Tabanbükü village and its vicinity $\mathrm{N}$ $38^{\circ} 31^{\prime} \mathrm{E} 038^{\circ} 25^{\prime}, \mathrm{N} 38^{\circ} 32^{\prime} \mathrm{E} 038^{\circ} 25^{\prime}$, N 38 $33^{\prime}$ E038 25', 730-812 m, Altınuşağ 1 village and its vicinity $\mathrm{N} 38^{\circ} 32^{\prime} \mathrm{E} 038^{\circ} 27^{\prime}, \mathrm{N} 38^{\circ} 32^{\prime} \mathrm{E} 038^{\circ} 26^{\prime}, 800-950 \mathrm{~m}$, Bilaluşağ 1 village and its vicinity $\mathrm{N}$

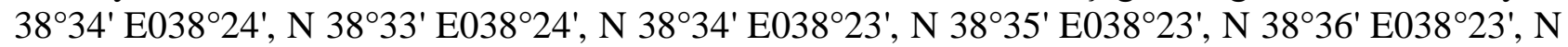

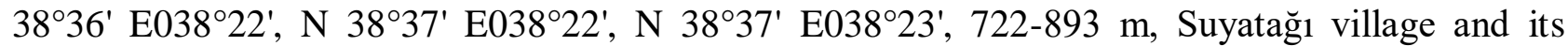
vicinity N $38^{\circ} 39^{\prime}$ E038 $24^{\prime}$, N $38^{\circ} 40^{\prime}$ E038 $25^{\prime}$, N $38^{\circ} 40^{\prime}$ E038 $24^{\circ}$ ', N $38^{\circ} 39^{\prime}$ E038 $25^{\prime}$, N $38^{\circ} 38^{\prime}$

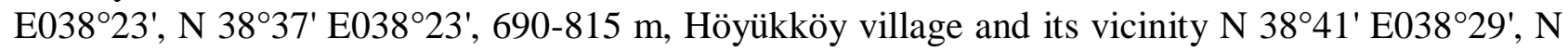
$38^{\circ} 40^{\prime} \mathrm{E} 038^{\circ} 29^{\prime}, \mathrm{N} 38^{\circ} 40^{\prime} \mathrm{E} 038^{\circ} 28^{\prime}, 709-740 \mathrm{~m}$, Kumlutarla village and its vicinity N $38^{\circ} 42^{\prime}$ $\mathrm{E} 038^{\circ} 30^{\prime}, \mathrm{N} 38^{\circ} 43^{\prime} \mathrm{E} 038^{\circ} 29^{\prime}, 702-750 \mathrm{~m}$ ). It was determined that $H$. salicifolium, which is its mycorrhiza, grows naturally from March to May ( Table 1, Fig. 2).

$P$. lefebvrei samples that were collected in the Elazı - Malatya area were analysed in detail, both macroscopically and microscopically (ascocarp, peridium, gleba, host plant, hymenium, hyphae, ascus and ascospore), and the results are presented in Fig. 2. 


\section{Picoa juniperi Vittad.}

Malatya city Battalgazi district (are found in the areas surrounding Meydancik village and its vicinity N $38^{\circ} 25^{\prime}$ E038 37', N 38 $25^{\prime}$ E038 $38^{\prime}$, N 38 $24^{\circ}$ E038 $38^{\prime}, 782-895$ m) seen in Table 1.

Elazı̆ city Baskil district (are found in the areas surrounding Pınarlı village and its vicinity

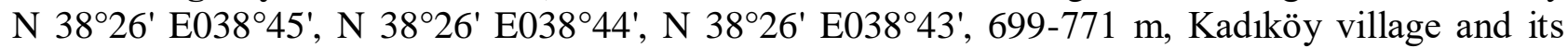

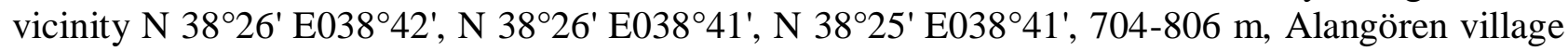

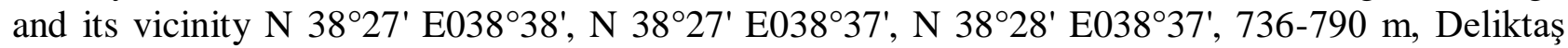

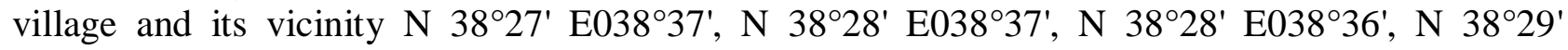
E038 $37^{\prime}$, N $38^{\circ} 29^{\prime}$ E038 $38^{\circ}$, N 38 ${ }^{\circ} 29^{\prime}$ E038 $39^{\prime}$, 762-1353 m, Gemici village and its vicinity N

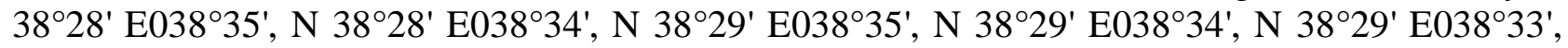
$759-818 \mathrm{~m}$, Hacimehmetli village and its vicinity N 38 $30^{\prime}$ E038 $32^{\prime}$, N 38 $30^{\prime}$ E038 $31^{\prime}$, N $38^{\circ} 29^{\prime}$ E038 $31^{\prime}$, N $38^{\circ} 29^{\prime}$ E038 32', 728-790 m, Çiğgdemlik village, Haciyusuflar hamlet and its vicinity

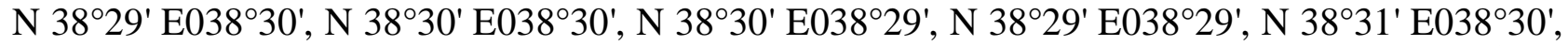

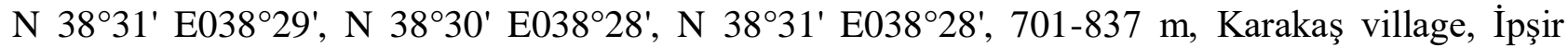

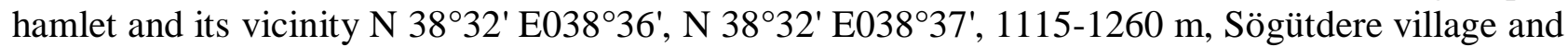
its vicinity $\mathrm{N} 38^{\circ} 31^{\prime} \mathrm{E} 038^{\circ} 41^{\prime}, \mathrm{N} 38^{\circ} 32^{\prime}$ E038 $41^{\prime}, 1174-1210 \mathrm{~m}$, Harabekayış village and its

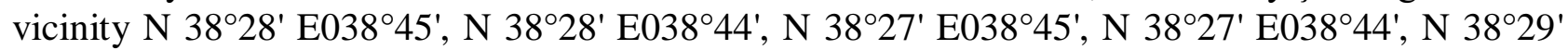
E038 45', N 38 30' E038 44', N 38 30' E038 45', 900-1200 m, İmikuşağ village and its vicinity N $38^{\circ} 31^{\prime} \mathrm{E} 038^{\circ} 27^{\prime}, \mathrm{N} 38^{\circ} 30^{\prime} \mathrm{E} 038^{\circ} 28^{\prime}, 724-812 \mathrm{~m}$, Tabanbükü village and its vicinity $\mathrm{N} 38^{\circ} 31^{\prime}$ $\mathrm{E} 038^{\circ} 25^{\prime}, \mathrm{N} 38^{\circ} 32^{\prime} \mathrm{E} 038^{\circ} 25^{\prime}, \mathrm{N} 38^{\circ} 33^{\prime} \mathrm{E} 038^{\circ} 25^{\prime}$, 730-812 m, Altınuşağ 1 village and its vicinity $\mathrm{N}$ $38^{\circ} 32^{\prime}$ E038 ${ }^{\circ} 27^{\prime}, \mathrm{N} 38^{\circ} 32^{\prime} \mathrm{E} 038^{\circ} 26^{\prime}$, 800-950 m, Bilaluşağ 1 village and its vicinity N $38^{\circ} 34^{\prime}$

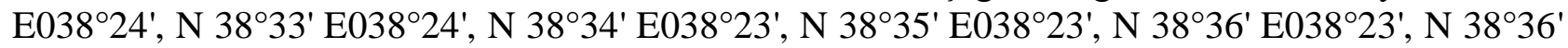

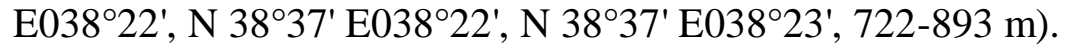

$P$. juniperi samples that were collected in the Elazığ - Malatya area were analysed in detail, both macroscopically and microscopically (ascocarp, peridium, gleba, host plants, ascus and ascospore), and the results are presented in Fig. 3.

\section{Pezizaceae Dumort.}

Terfezia (Tul. \& C.Tul) Tul. \& C.Tul.

\section{Terfezia boudieri Chatin}

Malatya city Central district (are found in the areas surrounding Kapıkaya village and its

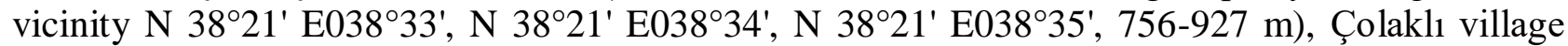

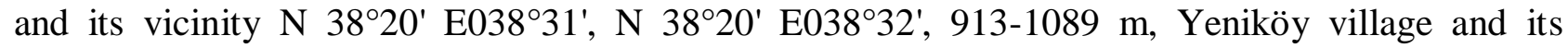

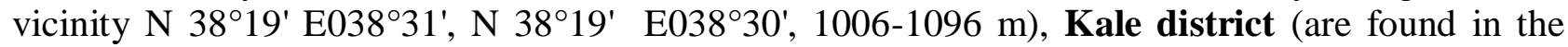

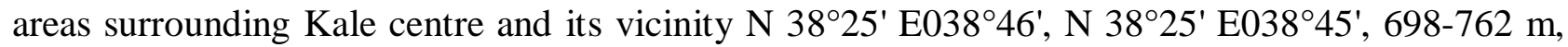

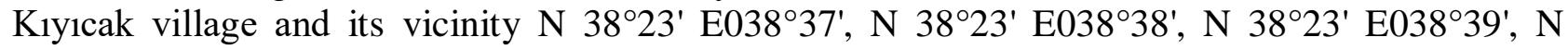
$38^{\circ} 24^{\prime}$ E038 $38^{\circ}, 729-886 \mathrm{~m}$ ), Battalgazi district (are found in the areas surrounding Meydanc1k village and its vicinity $\mathrm{N} 38^{\circ} 25^{\prime} \mathrm{E} 038^{\circ} 37^{\prime}, \mathrm{N} 38^{\circ} 25^{\prime} \mathrm{E} 038^{\circ} 38^{\prime}, \mathrm{N} 38^{\circ} 24^{\prime} \mathrm{E} 038^{\circ} 38^{\prime}, 782-895 \mathrm{~m}$,

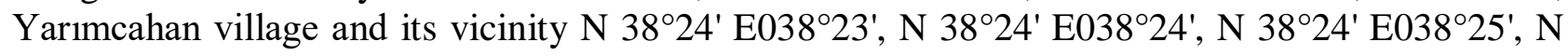

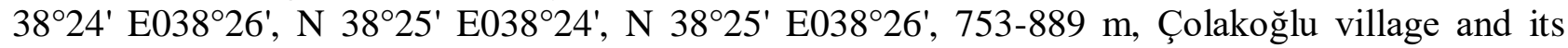

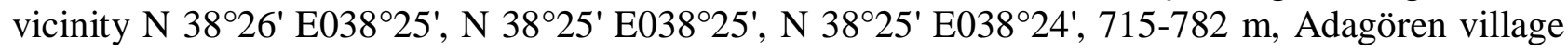
and its vicinity N $38^{\circ} 26^{\prime} \mathrm{E} 038^{\circ} 27^{\prime}, \mathrm{N} 38^{\circ} 26^{\prime} \mathrm{E} 038^{\circ} 28^{\prime}, 706-763 \mathrm{~m}$, Ağ 1 lyazı village and its vicinity

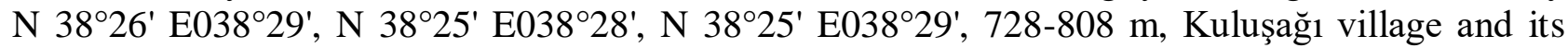

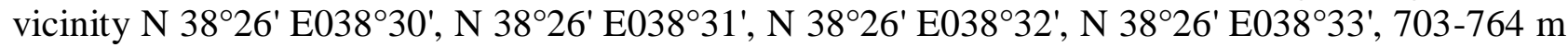

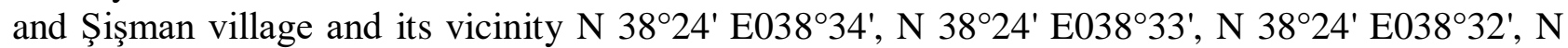

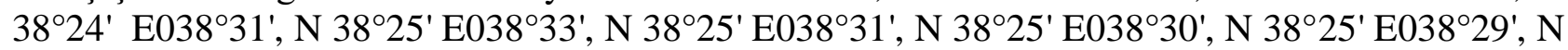
$38^{\circ} 25^{\prime} \mathrm{E} 038^{\circ} 28^{\prime}, 748-881 \mathrm{~m}$ ) and Arguvan district (are found in the areas surrounding Gümüşlü village and its vicinity N 38 $39^{\prime}$ E038 $22^{\prime}$, N 38 $39^{\prime}$ E038 $21^{\prime}, 705-762$ m, Morhamam village and its vicinity $\mathrm{N} 38^{\circ} 41^{\prime} \mathrm{E} 038^{\circ} 22^{\prime}, \mathrm{N} 38^{\circ} 40^{\prime} \mathrm{E} 038^{\circ} 23^{\prime}$, 712-754 m, Karababa village and its vicinity $\mathrm{N}$ $38^{\circ} 38^{\prime} \mathrm{E} 038^{\circ} 20^{\prime}, \mathrm{N} 38^{\circ} 38^{\prime} \mathrm{E} 038^{\circ} 19^{\prime}, 719-746 \mathrm{~m}$ ). It was determined that $H$. salicifolium, which is its mycorrhiza, grows naturally from March to May (rarely continue until mid-July) (Table 1, Fig. 4). 


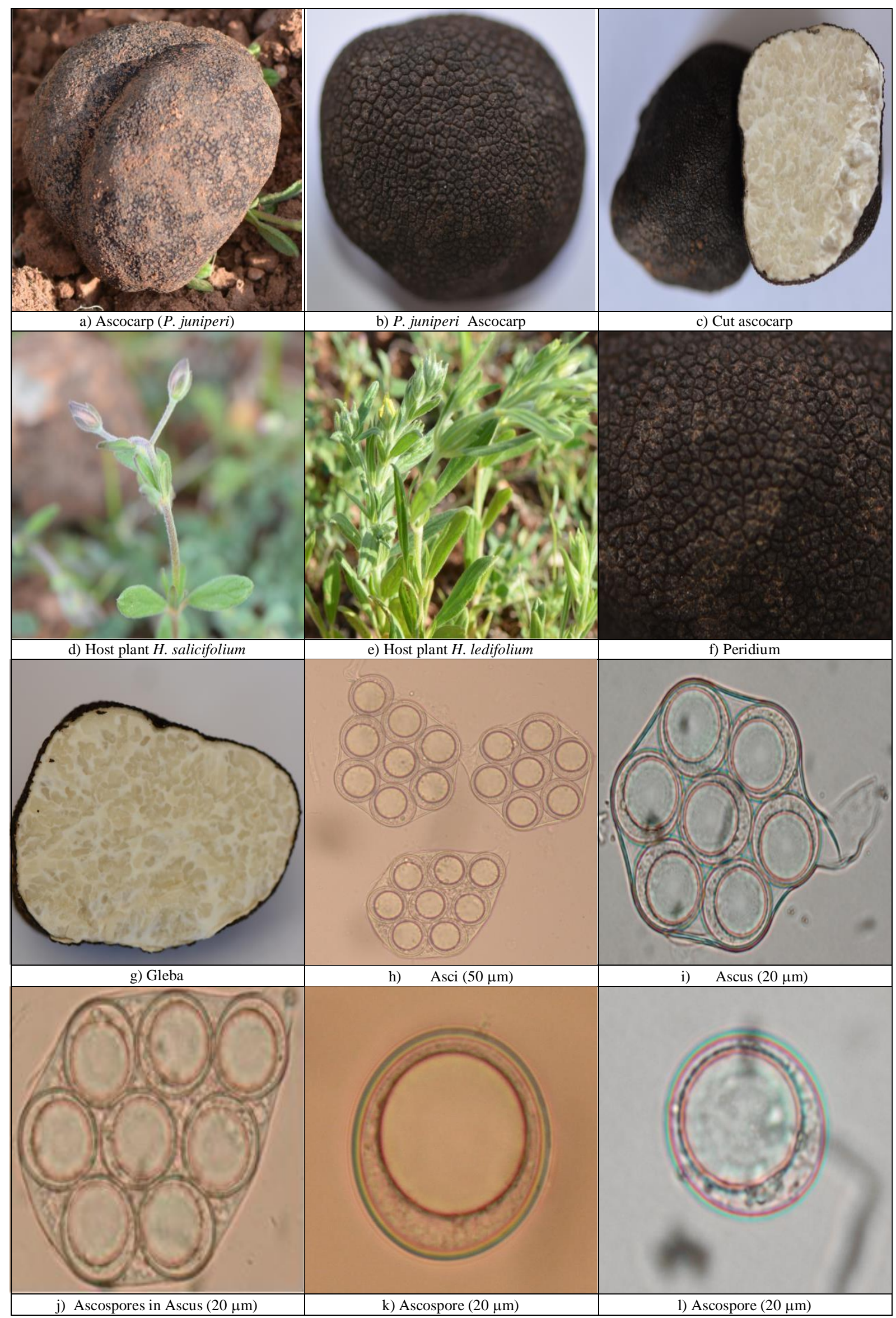

Fig. 3 - The macroscopic and microscopic characteristics (ascocarp, host plant, peridium, gleba, ascus and ascospores) of $P$. juniperi that were found in the Elazığ - Malatya area. 
It was determined that mostly $H$. salicifolium and rarely $H$. ledifolium which are its mycorrhiza, grows naturally from March to May (Table 1, Fig. 3).

Elazığ city Baskil district (are found in the areas surrounding Topaluşağ1 village and its

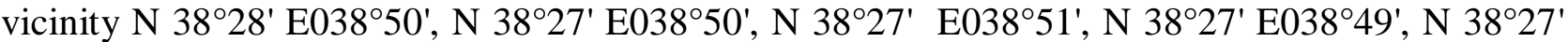

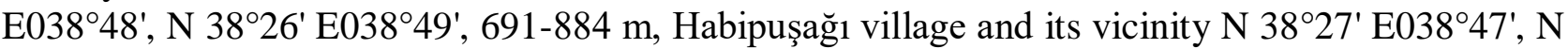
$38^{\circ} 26^{\prime}$ E038 $47^{\prime}$, N $38^{\circ} 26^{\prime}$ E038 $46^{\prime}$, N 38 $26^{\prime}$ E038 $45^{\prime}$ ', 696-768 m, P1narlı village and its vicinity

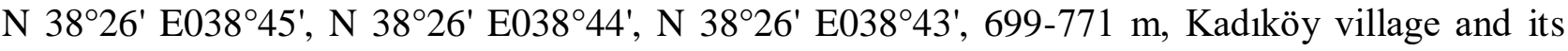

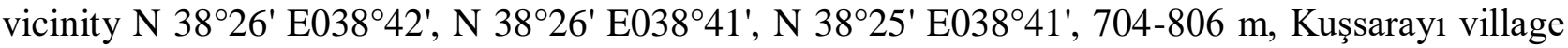

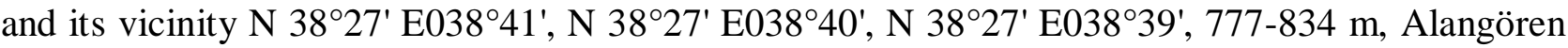

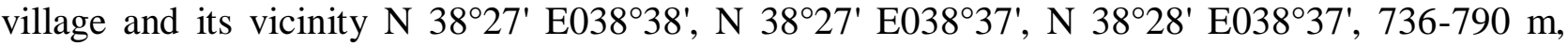

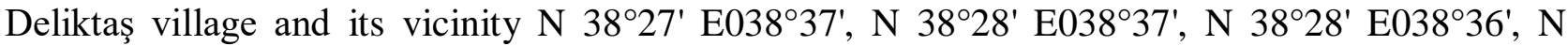

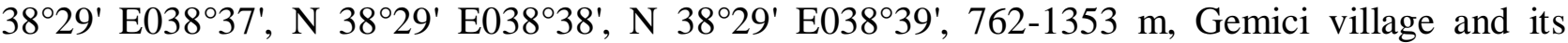

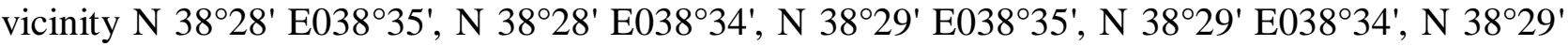
E038 $33^{\prime}, 759-818 \mathrm{~m}$, Konac1k village, Haydarkulu hamlet and its vicinity N $38^{\circ} 31^{\prime}$ E038 $33^{\circ}$, N

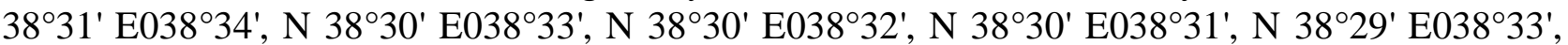

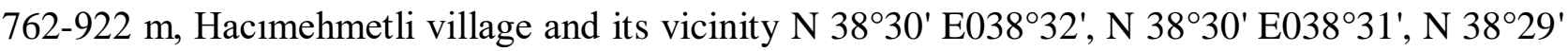
E038 $31^{\prime}$, N $38^{\circ} 29^{\prime}$ E038 $32^{\prime}, 728-790 \mathrm{~m}$, Çiğdemlik village, Haciyusuflar hamlet and its vicinity

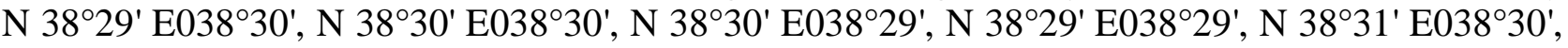

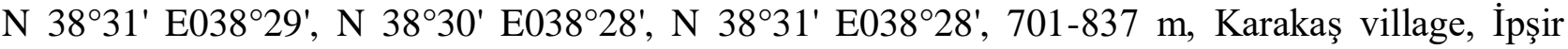
hamlet and its vicinity N 38 $32^{\prime}$ E038 $36^{\prime}$, N 38 $32^{\prime}$ E038 $37^{\prime}, 1115-1260 \mathrm{~m}$, Kızıluşağ 1 village and its vicinity N 38 $34^{\prime}$ E038 $40^{\prime}$, N 38 $34^{\prime}$ E038 $41^{\prime}$ 1303-1356 m, Yalındam village and its vicinity

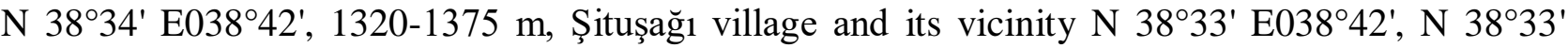
E038 $41^{\prime}, 1251-1351 \mathrm{~m}$, Sögütdere village and it vicinity N 38 $31^{\prime}$ E038 $41^{\circ}$, N $38^{\circ} 32^{\prime}$ E038 $41^{\prime}$, 1174-1210 m Harabekayış village and its vicinity N 38 $28^{\prime}$ E038 $45^{\circ}$ ', N 38 $28^{\prime}$ E038 $44^{\circ}$ ', N $38^{\circ} 27^{\prime}$

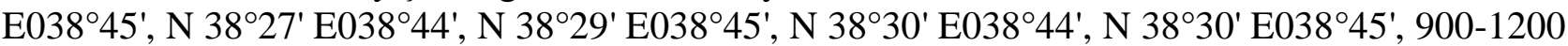

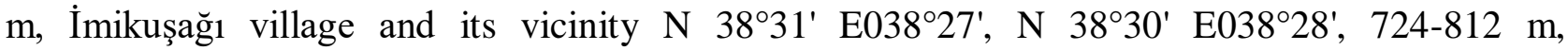
Tabanbükü village and its vicinity N $38^{\circ} 31^{\prime} \mathrm{E} 038^{\circ} 25^{\prime}$, N $38^{\circ} 32^{\prime} \mathrm{E} 038^{\circ} 25^{\prime}$, N $38^{\circ} 33^{\prime} \mathrm{E} 038^{\circ} 25^{\prime}$, 730 -

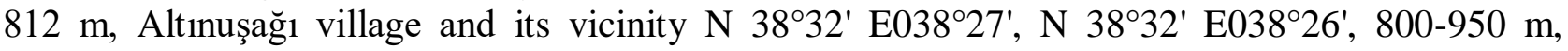

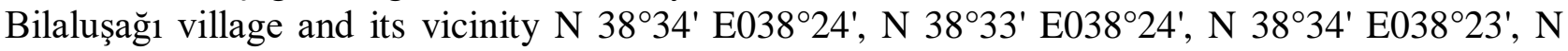

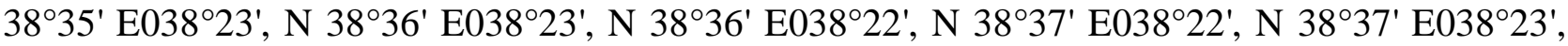

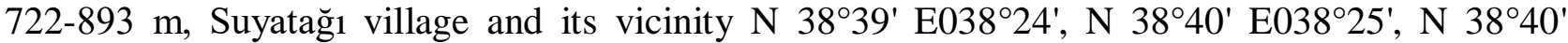

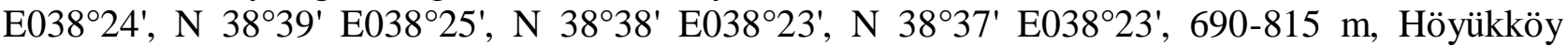

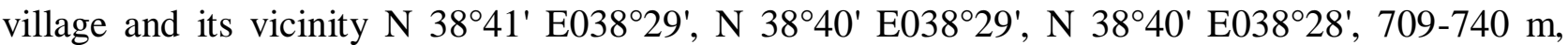
Kumlutarla village and its vicinity N 38 $42^{\prime}$ E038 $30^{\prime}$, N 38 $43^{\prime}$ E038 ${ }^{\circ} 29^{\prime}$, 702-750 m). It was determined that $H$. salicifolium, which is its mycorrhiza, grows naturally from March to May (rarely continue until mid-July) (Table 1, Fig. 4).

T. boudieri samples that were collected in the Elazı̆ - Malatya area were analysed in detail, both macroscopically and microscopically (ascocarp, peridium, gleba, host plant, hymenium, ascus and ascospore), and the results are presented in Fig. 4.

\section{Terfezia claveryi Chatin}

Elazığ city Baskil district (are found in the areas surrounding Gemici village and its vicinity

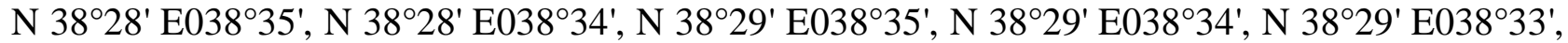

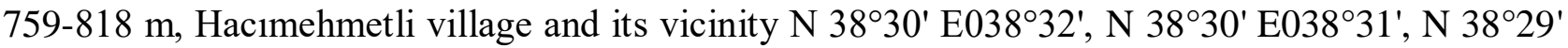
E038 $31^{\prime}$, N 38 $29^{\prime}$ E038 32', 728-790 m, Çiğdemlik village, Haciyusuflar hamlet and its vicinity

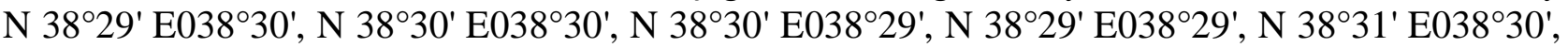

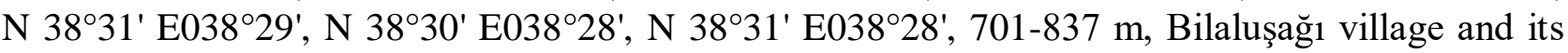

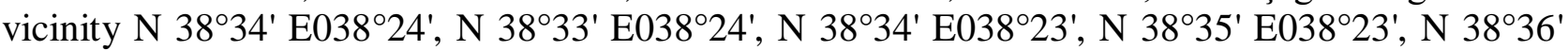

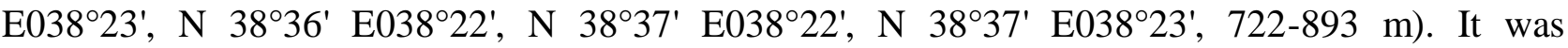
determined that $H$. salicifolium, which is its mycorrhiza, grows naturally from March to May (Table 1, Fig. 5). 


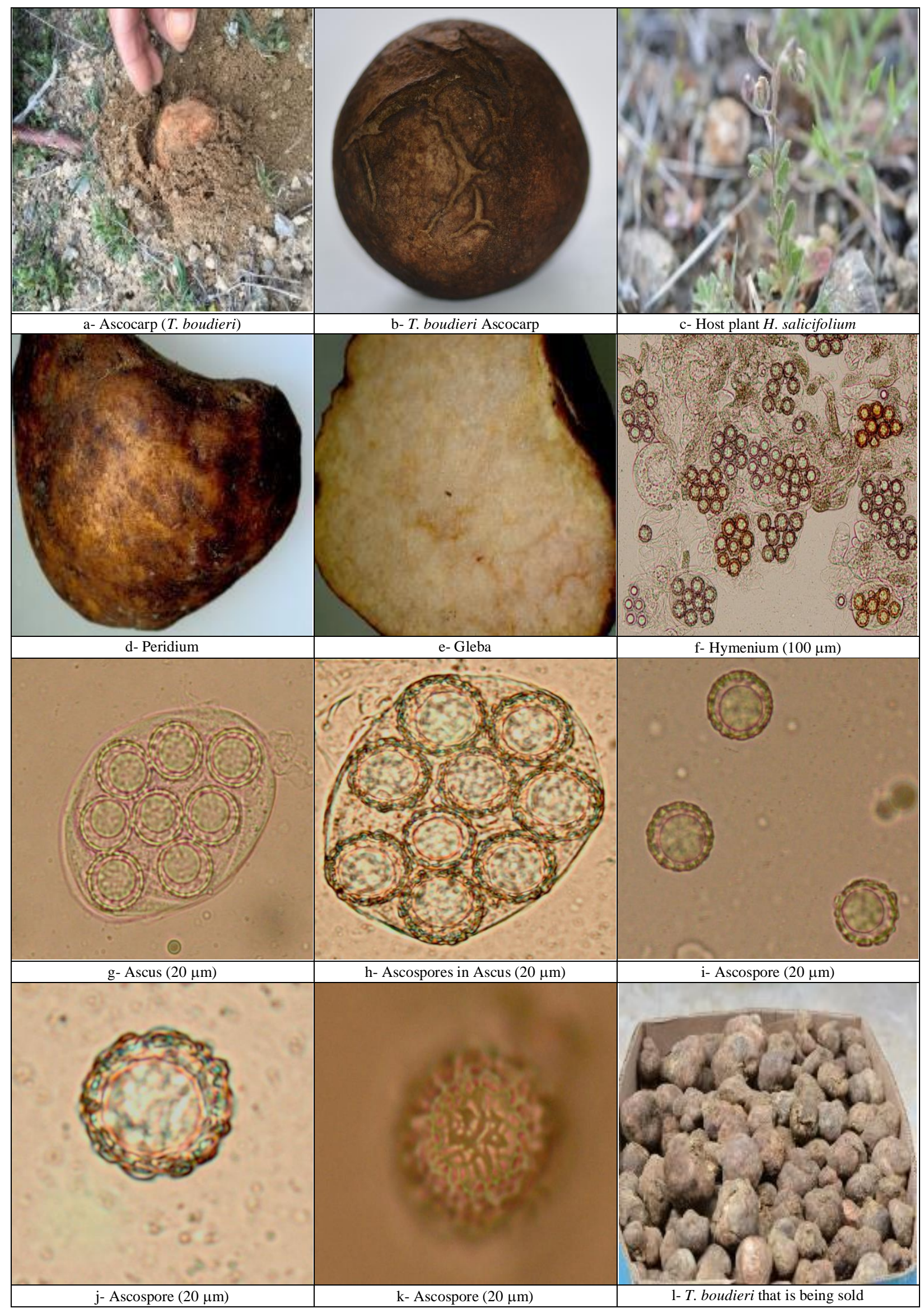

Fig. 4 - The macroscopic and microscopic characteristics (ascocarp, host plant, peridium, gleba, hyphae, hymenium, ascus and ascospore) of T. boudieri that were found in the Elazığ - Malatya area. 
T. claveryi samples that were collected in the Elazığ area were analysed in detail, both macroscopically and microscopically (ascocarp, peridium, gleba, host plant, hymenium, ascus and ascospore), and the results are presented in Fig. 5.

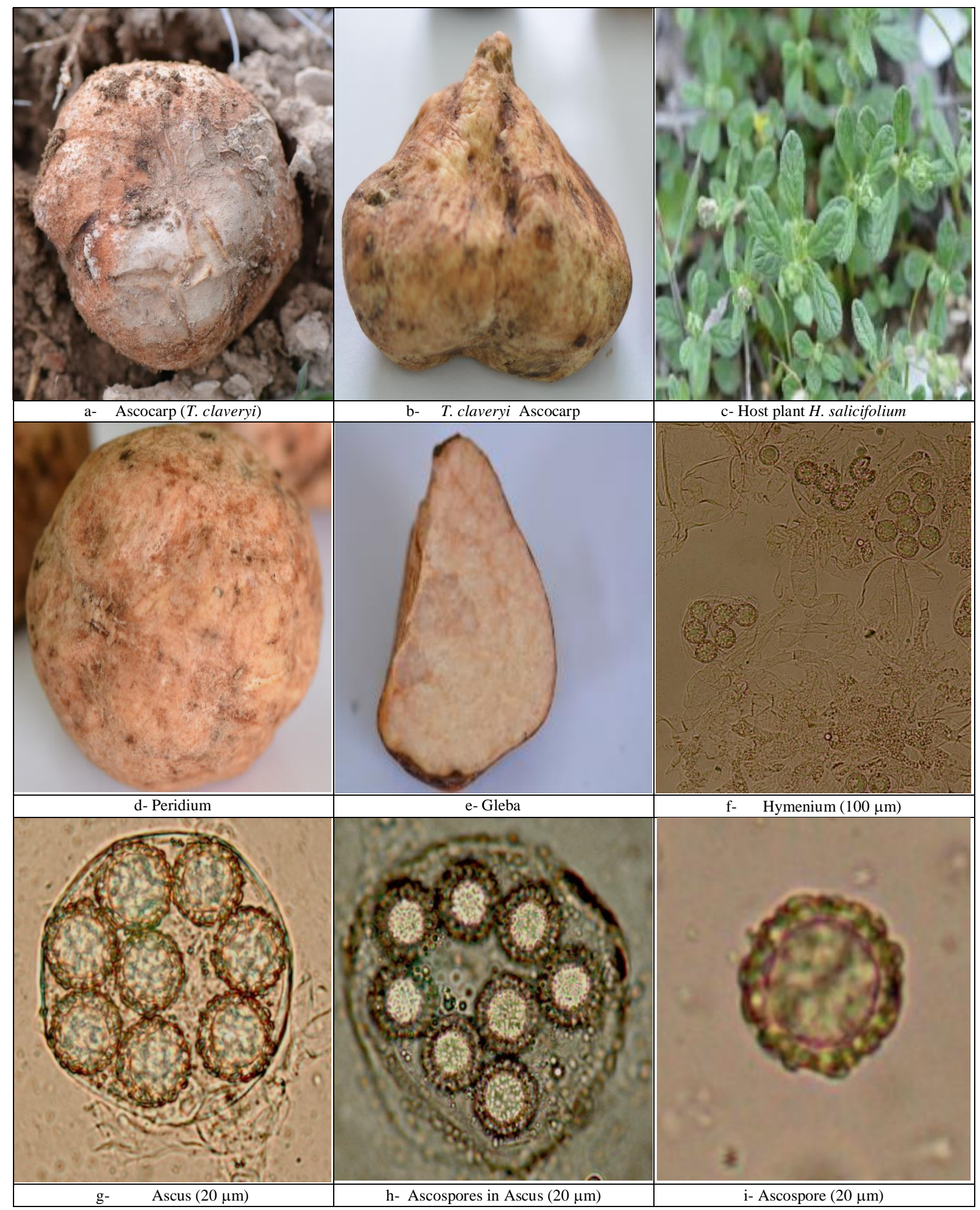

Fig. 5 - The macroscopic and microscopic characteristics (ascocarp, host plant, peridium, gleba, hymenium, ascus and ascospore) of $T$. claveryi that were found in the Elazıg area. 


\section{Terfezia olbiensis Tulasne \& C.Tulasne}

Malatya city Kale district (are found in the areas surrounding Kiyıcak village and its

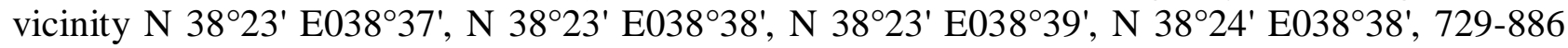
$\mathrm{m}$ ), Battalgazi district (are found in the areas surrounding Meydanc1k village and its vicinity $\mathrm{N}$ $38^{\circ} 25^{\prime} \mathrm{E} 038^{\circ} 37^{\prime}, \mathrm{N} 38^{\circ} 25^{\prime} \mathrm{E} 038^{\circ} 38^{\prime}, \mathrm{N} 38^{\circ} 24^{\prime}$ E038 $38^{\prime}$, 782-895 m, Yarımcahan village and its

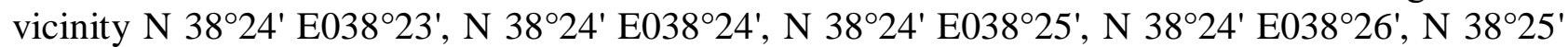
E038 $24^{\prime}, N^{\circ} 38^{\circ} 25^{\prime} \mathrm{E} 038^{\circ} 26^{\prime}, 753-889 \mathrm{~m}$, Çolakoğlu village and its vicinity N $38^{\circ} 26^{\prime} \mathrm{E} 038^{\circ} 25^{\prime}, \mathrm{N}$ $38^{\circ} 25^{\prime} \mathrm{E} 038^{\circ} 25^{\prime}, \mathrm{N} 38^{\circ} 25^{\prime} \mathrm{E} 038^{\circ} 24^{\prime}, 715-782 \mathrm{~m}$, Adagören village and its vicinity N $38^{\circ} 26^{\prime}$

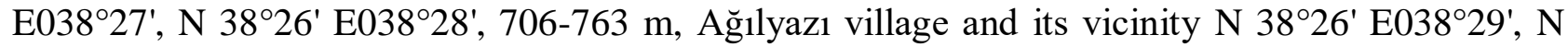
$38^{\circ} 25^{\prime} \mathrm{E} 038^{\circ} 28^{\prime}, \mathrm{N} 38^{\circ} 25^{\prime} \mathrm{E} 038^{\circ} 29^{\prime}, 728-808 \mathrm{~m}$, Kuluşağ 1 village and its vicinity N $38^{\circ} 26^{\prime}$

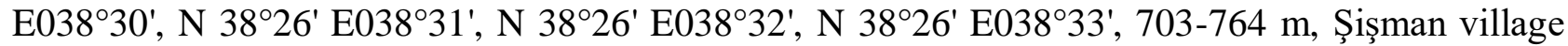
and its vicinity $\mathrm{N} 38^{\circ} 24^{\prime} \mathrm{E} 038^{\circ} 34^{\prime}, \mathrm{N} 38^{\circ} 24^{\prime} \mathrm{E} 038^{\circ} 33^{\prime}, \mathrm{N} 38^{\circ} 24^{\prime} \mathrm{E} 038^{\circ} 32^{\prime}, \mathrm{N} 38^{\circ} 24^{\prime} \mathrm{E} 038^{\circ} 31^{\prime}, \mathrm{N}$ $38^{\circ} 25^{\prime} \mathrm{E} 038^{\circ} 33^{\prime}, \mathrm{N} 38^{\circ} 25^{\prime} \mathrm{E} 038^{\circ} 31^{\prime}, \mathrm{N} 38^{\circ} 25^{\prime} \mathrm{E} 038^{\circ} 30^{\prime}$, N 38 $25^{\prime} \mathrm{E} 038^{\circ} 29^{\prime}$, N 38 $25^{\prime}$ E038 $28^{\prime}$, $748-881 \mathrm{~m})$. It was determined that $H$. salicifolium, which is its mycorrhiza, grows naturally from March to May (Table 1, Fig. 6).

Elaž $\breve{g}$ city Baskil district (are found in the areas surrounding Pınarlı village and its vicinity

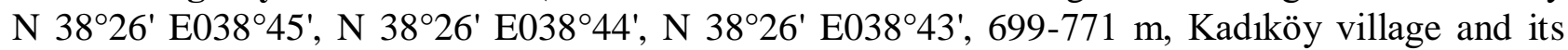

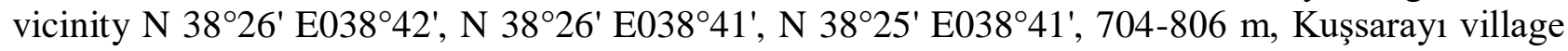
and its vicinity $\mathrm{N} 38^{\circ} 27^{\prime} \mathrm{E} 038^{\circ} 41^{\prime}, \mathrm{N} 38^{\circ} 27^{\prime} \mathrm{E} 038^{\circ} 40^{\prime}$, N 38 $27^{\circ}$ E038 $38^{\circ}$ ', 777-834 m, Alangören

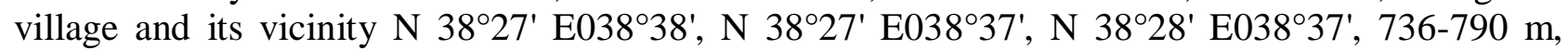

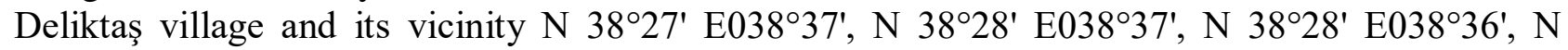
$38^{\circ} 29^{\prime} \mathrm{E} 038^{\circ} 37^{\prime}, \mathrm{N} 38^{\circ} 29^{\prime} \mathrm{E} 038^{\circ} 38^{\prime}, \mathrm{N} 38^{\circ} 29^{\prime} \mathrm{E} 038^{\circ} 39^{\prime}, 762-1353 \mathrm{~m}$, Gemici village and its

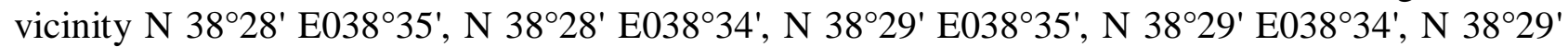
E038 33', 759-818 m, Konacik village, Haydarkulu hamlet and its vicinity N 38 $31^{\prime}$ E038 $33^{\circ}$, N

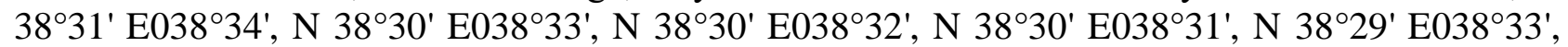

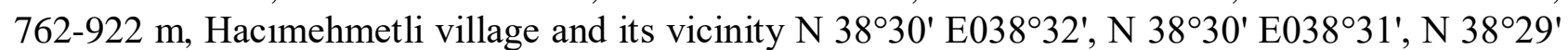
E038 $31^{\prime}, N_{3}^{\circ} 29^{\prime}$ E038 $32^{\prime}, 728-790$ m, Çiğdemlik village, Haciyusuflar hamlet and its vicinity

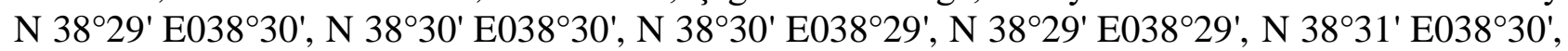

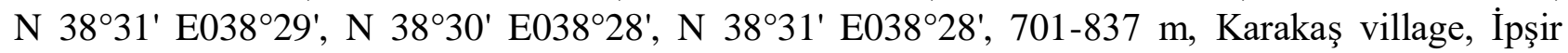

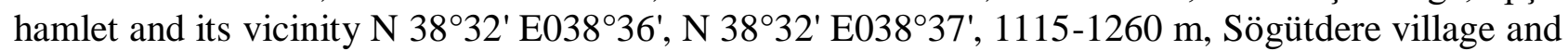
its vicinity $\mathrm{N} 38^{\circ} 31^{\prime} \mathrm{E} 038^{\circ} 41^{\prime}, \mathrm{N} 38^{\circ} 32^{\prime} \mathrm{E} 038^{\circ} 41^{\prime}, 1174-1210 \mathrm{~m}$, Harabekayış village and its

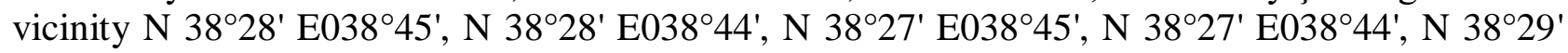
E038 $45^{\prime}$, N 38 30' E038 44', N 38 30' E038 $45^{\circ}$ ', 900-1200 m, İmikuşağ 1 village and its vicinity N $38^{\circ} 31^{\prime} \mathrm{E} 038^{\circ} 27^{\prime}, \mathrm{N} 38^{\circ} 30^{\prime} \mathrm{E} 038^{\circ} 28^{\prime}, 724-812 \mathrm{~m}$, Tabanbükü village and its vicinity N $38^{\circ} 31^{\prime}$ E038 $25^{\prime}, \mathrm{N} 38^{\circ} 32^{\prime} \mathrm{E} 038^{\circ} 25^{\prime}, \mathrm{N} 38^{\circ} 33^{\prime} \mathrm{E} 038^{\circ} 25^{\prime}$, 730-812 m, Altınuşağ 1 village and its vicinity N $38^{\circ} 32^{\prime} \mathrm{E} 038^{\circ} 27^{\prime}, \mathrm{N} 38^{\circ} 32^{\prime} \mathrm{E} 038^{\circ} 26^{\prime}, 800-950 \mathrm{~m}$, Bilaluşağ 1 village and its vicinity N $38^{\circ} 34^{\prime}$

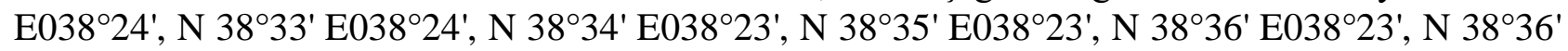
E038 $\left.22^{\prime}, N 38^{\circ} 37^{\prime} \mathrm{E} 038^{\circ} 22^{\prime}, \mathrm{N} 38^{\circ} 37^{\prime} \mathrm{E} 038^{\circ} 23^{\prime}, 722-893 \mathrm{~m}\right)$. It was determined that $H$. salicifolium, which is its mycorrhiza, grows naturally from March to May (Table 1, Fig. 6).

T. olbiensis samples that were collected in the Elazı $\breve{g}$ - Malatya area were analysed in detail, both macroscopically and microscopically (ascocarp, peridium, gleba, host plant, hymenium, ascus and ascospore), and the results are presented in Fig. 6.

\section{Discussion}

Picoa lefebvrei, P. juniperi, Terfezia boudieri, T. claveryi and T. olbiensis were collected from the field during our work. The morphological, ecological characteristics of the truffles, and the characteristics of the areas they grow were documented (Table 1). The required macroscopic and micrsocopic data were gathered using standard micological techniques (Trappe 1979, Alsheikh 1994, Montecchi \& Sarasini 2000, Ferdman et al. 2005, Castellano \& Türkoğlu 2012, Türkoğlu \& Castellano 2014). The data, which was collected after the fieldwork and laboratory studies, were evaluated, the species were defined using the relevant literary sources (Alsheikh \& Trappe 1983, Gücin 1983, Alsheikh 1994, Moreno et al. 2000, Montecchi \& Sarasini 2000, Kirk et al. 2008, 


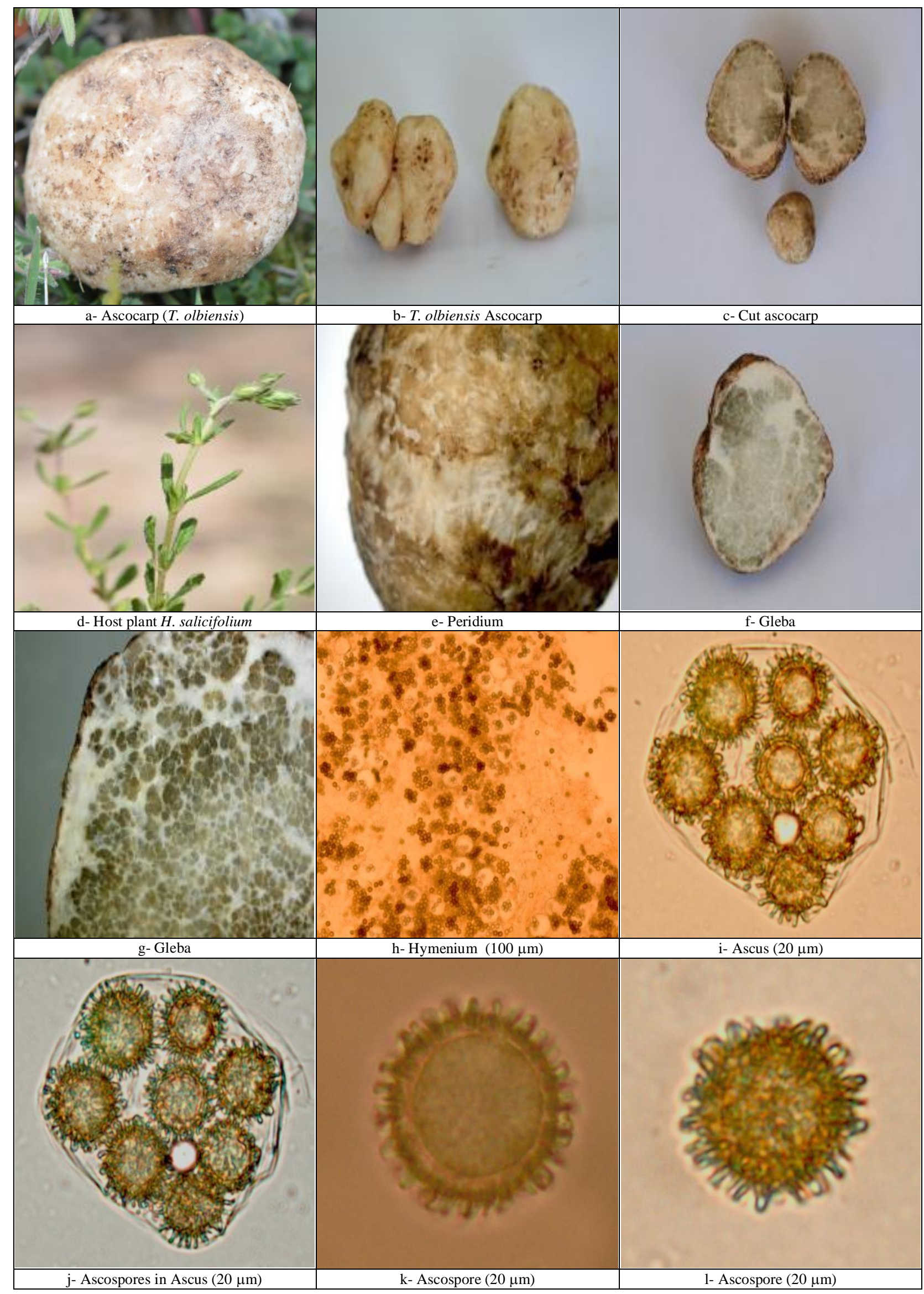

Fig. 6 - The macroscopic and microscopic characteristics (ascocarp, host plant, peridium, gleba, hyphae, hymenium, ascus and ascospore) of T. olbiensis that were found in the Elazığ - Malatya area. 
Kagan-Zur et al. 2014), and the definitions were verified based on the works of Gücin (1983), Gücin et al. (2010), Türkoğlu \& Castellano (2014), and Türkoğlu et al. (2015).

Picoa lefebvrei grows naturally at Elazığ, Şanlıurfa, Denizli, Aksaray and Konya (Gücin et al. 2010, Şahin 2012, Türkoğlu et al. 2015), P. juniperi grows naturally at Kayseri, Uşak, Denizli, Nevşehir, Konya, Elazığ, Afyonkarahisar and Antalya (Türkoğlu \& Yağız 2012, Türkoğlu \& Castellano 2014, Türkoğlu et al. 2015), Terfezia arenaria grows naturally at Aydın, Isparta, Malatya and Konya (Öder 1988, Işıloğlu \& Öder 1995, Afyon 1996, Kaşık et al. 1998, Türkoğlu et al. 2015), T. claveryi grows naturally at Denizli, Şanlıurfa, Konya, Aksaray, Diyarbakır, Karaman, Yozgat, Konya and Kastamonu (Bekçi et al. 2011, Şahin 2012, Türkoğlu et al. 2015), T. leptoderma grows naturally at Uşak and Denizli (Castellano \& Türkoğlu 2012; Türkoğlu \& Castellano 2014), T. olbiensis grows naturally at Uşak, Konya and Nevşehir (Türkoğlu \& Castellano 2014), T. boudieri grows naturally at Uşak, Batman, Karaman, Elazı̆̆, Malatya, Eskişehir, Mardin, Şanlıurfa, Diyarbakır, Niğde, Gaziantep, Konya, Ankara, Kutahya, Aksaray, Kırşehir etc. (Gücin 1983, Gücin 1990, Gücin \& Dülger 1997, Kaşık et al. 2001, Doğan \& Öztürk 2006, Demir et al. 2007, Aydın 2009, Akyüz et al. 2012, Şahin 2012, Türkoğlu \& Yağız 2012, Yamaç 2012 etc.), which are arid, semi-arid truffle types. It was determined that $P$. lefebvrei, $P$. juniperi, T. olbiensis, T. claveryi and T. boudieri (Figure 2-6) grow naturally at the area, where the Malatya (Merkez, Kale, Battalgazi, Arguvan districts and their vicinities) and Elazı̆̆ (Baskil district and its vicinity) provincial borders intersect, together with the area around Kömürhan Bridge, and at areas along the shores of the Karakaya Dam, as seen in Table 1 and Fig. 1.

Desert truffle types grow at parts of the Mediterranean region with arid or semi arid conditions, and represented by types including Terfezia, Tirmania and Picoa. These exist as mycorrhizae with the roots of plant types of the Cistacaea family, of which various species of the Helianthemum (L.) genus are a part of, and which exist in areas where different soil characteristics can be observed (Diez et al. 2002, Gutierrez et al. 2003, Mandeel \& Al-Laith 2007, Kagan-Zur \& Roth-Bejerano 2008, Morte et al. 2008, Kovacs et al. 2011, Chevalier 2014, Kagan-Zur et al. 2014, Roth-Bejerano et al. 2014). It was determined that Picoa lefebvrei, P. juniperi, Terfezia boudieri, $T$. claveryi and T. olbiensis naturally grow usually at areas where Helianthemum salicifolium, and rarely at areas where Helianthemum ledifolium exist within the study area (Table 1, Fig. 2d, 3d-e, $4 \mathrm{c}, 5 \mathrm{c}$, and 6d).

Picoa lefebvrei has a pale yellow-brown to yellow-brown peridial surface with scattered to numerous, irregular warts, while $P$. juniperi has a brown-black to black peridial surface with regularly arranged and uniformly distributed warts (Türkoğlu et al. 2015). Terfezia olbiensis has small ascocarps with pale colours and also fairly short spores ornamented with narrow to broadly truncate cones. It appears to be the most uncommon of the Terfezia species found in Turkey. Montecchi and Sarasini (2000) reported T. olbiensis from under Quercus or Pinus spp., but our specimens and examples found by some researchers were found in sandy soils associated with Helianthemum spp. (Türkoğlu \& Castellano 2014). Terfezia claveryi has an off-white peridial surface at first but later has a red-brown, finally dark red brown or black-brown peridial surface. It has a pale yellow gleba with distinct sterile veins. It has spores ornamented with a prominent irregular reticulation with irregular alveolae (Türkoğlu et al. 2015).

Terfezia boudieri is a species close to T. claveryi, and some authors consider them synonyms. In any case, their macro-morphological nature, ecology, hosts, fruiting periods, etc. are extremely similar. The greatest morphometric diffence is noted at a microscopic level and lies in spore ornamentation. In $T$. claveryi, the spore presents a well-defined reticulum upon which slightly prominet warts might appear in accordance with the degree of maturity, whereas pronounced and sometimes truncated warts appear in the spores of $T$. boudieri, which resemble those of $T$. arenaria. This character is insufficient to separate both species because of a possible continuum between the ornamentation of one species and that of the other, given the presence of verrucose growths on the reticulum nodes of the T. claveryi spores (Morte et al. 2009). In some parts of Elaziğ and Malatya, it is quite possible to find carpophores of T. boudieri mixed with those of $T$. claveryi since even the rural population cannot distinguish between the two species. 
It was observed that in all research areas, Terfezia boudieri and Picoa lefebvrei were found in the same growing areas, and at some areas both Terfezia and Picoa species were found together (Table 1). It is highly possible that with more fieldwork and longterm research, other types of these species can also be found in these areas. In conclusion, we report the first records of two Terfezia species (T. olbiensis and T. claveryi) in the research field, and that we determined new areas for Terfezia boudieri, Picoa juniperi and P. lefebvrei in the Malatya and Elazı̆g provinces. Furthermore, the habitats of these species should be protected by turning the growing fields into natural protected areas, in order to assure that new generations can also exploit these semi-arid truffles, which grow at a certain location only during a certain part of the year, and consumed by the locals as a daily source of nutrition.

\section{Acknowledgements}

We would like to thank The Scientific and Technical Research Council of Turkey (TÜBITAK) for financially supporting this project (TOVAG-114O065). We wish to express our sincere gratitude to Dr. Aziz Türkoğlu (Muğla Sitkı Koçman University, Turkey), Dr. Varda Kagan Zur (Ben Gurion University, Israel), and Dr. T. Emre Şerifoğlu (Bitlis Eren University, Turkey) for their valuable comments and suggestions.

\section{References}

Afyon A. 1996 - Some macrofungi determined of Isparta province in Turkey. Turkish Journal of Botany 20, 161-164.

Akyüz M, Kirbag S, Kursat M. 2012 - Ecological aspects of the arid and semi-arid truffle in Turkey: evaluation of soil characteristics, morphology, distribution, and mycorrhizal relationships. Turkish Journal of Botany 36, 386-391.

Alsheikh AM, Trappe JM. 1983 - Taxonomy of Phaeangium lefebvrei, a desert truffle eaten by birds. Canadian Journal of Botany 61, 1919-1925.

Alsheikh AM. 1994 - Taxonomy and mycorrhizal ecology of the desert truffles in the genus Terfezia. Doctoral Dissertation, Corvallis, Oregon State University, p 239.

Aydın S. 2009 - Terfezia boudieri Chatin ve Lactarius vellereus (Fr.) Fr.'un antioksidan, antimikrobiyal etkilerinin ve yağ asidi kompozisyonunun belirlenmesi. Yüksek Lisans Tezi, Selçuk Üniversitesi Fen Bilimleri Enstitüsü, Biyoloji Anabilim Dalı, Konya, s 119.

Bekçi H, Altınsoy B, Sarıkaya S, Onbasılı D, Çelik GY. 2011 - Antimicrobial activity of some macrofungi collected from Kastamonu province. Kastamonu University Journal of Forestry Faculty 11, 187-190.

Castellano MA, Türkoğlu A. 2012 - New records of truffle taxa in Tuber and Terfezia from Turkey. Turkish Journal of Botany 36, 295-298.

Chevalier G. 2014 - The European Desert Truffles. In: Kagan-Zur V, Roth-Bejerano N, Sitrit Y, Morte A, editors. Desert Truffles. Springer Berlin Heidelberg, pp. 121-141.

Demir S, Demirel K, Uzun Y. 2007 - Macrofungi of Batman province. Ekoloji 16, 37-42.

Diez J, Manjon JL, Martin F. 2002 - Molecular phylogeny of the mycorrhizal desert truffles (Terfezia and Tirmania), host specificity and edaphic tolerance. Mycologia 94, 247-259.

Doğan HH, Öztürk C. 2006 - Macrofungi and their distribution in Karaman province, Turkey. Turkish Journal of Botany 30, 193-207.

Ferdman Y, Aviram S, Roth-Bejerano N, Trappe JM, Kagan-Zur V. 2005 - Phylogenetic studies of Terfezia pfeilii and Choiromyces echinulatus (Pezizales) support new genera for Southern African truffles: Kalaharituber and Eremiomyces. Mycological Research 109, 237-245.

Gutiérrez A, Morte A, Honrubia M. 2003 - Morphological characterization of the mycorrhiza formed by Helianthemum almeriense Pau with Terfezia claveryi Chatin and Picoa lefebvrei (Pat.) Maire. Mycorrhiza 13, 299-307. 
Gücin F. 1983 - Elazığ ili sınırları içinde yetişen bazı makrofunguslar üzerinde taksonomik bir araştırma. Doktora Tezi, Ege Üniversitesi Fen Fakültesi Biyoloji Bölümü, İzmir.

Gücin F. 1990 - Macrofungi found surroundings of Elazı̆̆. Turkish Journal of Botany 14, 171-177.

Gücin F, Dülger B. 1997 - Yenen ve antimikrobiyal aktiviteleri olan keme mantarı (Terfezia boudieri Chatin) üzerinde araştırmalar. Ekoloji 23, 27 - 33.

Gücin F, Kaya A, Soylu MK, Uzun Y. 2010 - Picoa Vittad., a new truffl e genus record for Turkey. Biological Diversity and Conservation 3, 23-25.

Iş̧loğlu M, Oder N. 1995 - Macrofungi of Malatya province. Turkish Journal of Botany 19, 321-324.

Kagan-Zur V, Roth-Bejerano N. 2008 - Desert truffles. Fungi 1, 32-37.

Kagan-Zur V, Akyuz M. 2014 - Asian Mediterranean desert truffles. In: Kagan-Zur V, Roth-Bejerano N, Sitrit Y, Morte A editors. Desert Truffles. Springer Berlin Heidelberg, pp. 159-171.

Kagan-Zur V, Roth Bejerano N, Sitrit Y, Morte A. 2014 - Desert Truffle (phylogeny, physiology, distribution and domestication). Springer Verlag Berlin Heidelberg, p. 397.

Kaşık G, Ozturk C, Akkoz C, Doğan HH. 1998 - Some macrofungi determined in SU Alaaddin Keykubat campus. Selcuk Universitesi Fen Edebiyat Fakultesi Fen Dergisi 15, 87-99.

Kaşık G, Ozturk C, Toprak E. 2001 - Macrofungi of Niğde province (Turkey). Ot Sitematik Botanik Dergisi 8, 137-142.

Kaya A. 2009 - Macromycetes of Kahramanmaraş Province (Turkey). Mycotaxon 108, 31-38.

Kirk PM, Cannon PF, Minter DW, Stalfers JA. 2008 - Authors of Fungal Names [Index to Fungi Supplement]. Wallingford, UK: CABI Bioscience.

Kovács GM, Balázs TK, Calonge FD, Martín MP. 2011 - The diversity of Terfezia desert truffles: new species and a highly variable species complex with intrasporocarpic nrDNA ITS heterogeneity. Mycologia 103, 841-853.

Mandeel QA, Al-Laith AA. 2007 - Ethnomycological aspects of the desert truffle among native Bahraini and non-Bahraini peoples of the Kingdom of Bahrain. Journal of Ethnopharmacology 110, 118-129.

Montecchi A, Sarasini M. 2000 - Fungi ipogei d'Europa. Trento, Italy: Associazone Micologica Bresadola.

Moreno G, Diez J, Manjón JL. 2000 - Picoa lefebvrei and Tirmania nivea, two rare hypogeous fungi from Spain. Mycological Research 104, 378-381.

Moreno G, Alvarado P, Manjón JL. 2014 - Hypogeous Desert Fungi. In: Kagan-Zur V, RothBejerano N, Sitrit Y, Morte A editors, Desert Truffles. Springer Berlin Heidelberg, pp. 3-20.

Morte A, Honrubia M, Gutierrez A. 2008 - Biotechnology and cultivation of desert truffles. In: Varma A, editor. Mycorrhiza: State of the art, genetics and molecular biology, eco-function, biotechnology, eco-physiology, structure and systematics, Berlin, Heidelberg, Springer-Verlag, pp. 467-483.

Morte A, Zamora M, Gutiérrez A, Honrubia M. 2009 - Desert truffle cultivation in semiarid Mediterranean areas. In: Azcón-Aguilar C, Barea JM, Gianinazzi S, Gianinazzi-Pearson V, editors. Mycorrhizas: Functional Processes and Ecological Impact, Springer Berlin Heidelberg, pp. 221-233.

Öder N. 1988 - Taxonomic investigations of important edible and poisonous mushrooms growing in the Konya center and some Districts of Konya. Selçuk Universitesi Fen Edebiyat Fakültesi Dergisi 8, 237-257.

Roth-Bejerano N, Li YF, Kagan-Zur V. 2004 - Homokaryotic and heterokaryotic hyphae in Terfezia. Antonie Leeuwenhoek 85, 165-168. 
Roth-Bejerano N, Navarro-Ródenas A, Gutiérrez A, 2014 - Types of mycorrhizal association. In: Kagan-Zur V, Roth-Bejerano N, Sitrit Y, Morte A, editors. Desert Truffles. Springer Berlin Heidelberg, pp. 69-80.

Solak MH, Isıloğlu M, Kalmıs E, Allı H. 2007 - Macrofungi of Turkey. Checklist, 1, Universiteliler Ofset, Bornova / İzmir.

Şahin A. 2012 - Konya yöresinde yetişen Terfezia türleri ve etnomikolojik özellikleri. Yüksek Lisans Tezi, Selçuk Üniversitesi Fen Bilimleri Enstitüsü, Biyoloji Anabilim Dalı, Konya.

Trappe JM. 1979 - The orders, families, and genera of hypogeous Ascomycotina (truffles and their relatives. Mycotaxon 9, 297-340.

Türkoğlu A, Yağız D. 2012 - Contributions to the macrofungal diversity of Uşak Province. Turkish Journal of Botany 36, 580-589.

Türkoğlu A, Castellano MA. 2014 - New records of some Ascomycete truffle fungi from Turkey. Turkish Journal of Botany 38, 406-416.

Türkoğlu A, Castellano MA, Trappe JM, Güngör MY. 2015 - Turkish truffles I: 18 new records for Turkey. Turkish Journal of Botany 39, 359-376.

Yamaç M. 2012 - Eskişehir ili makrofungus çeşitliliği. Eski Yeni Aylık Şehir Kültür Dergisi 42, $5-10$. 